\title{
Research Article \\ Primitive Zero-Symmetric Sign Pattern Matrices with Zero Diagonal Attaining the Maximum Base
}

\author{
Ling Zhang, ${ }^{1}$ Ting-Zhu Huang, ${ }^{1}$ and Zhongshan $\mathrm{Li}^{2,3}$ \\ ${ }^{1}$ School of Mathematical Sciences, University of Electronic Science and Technology of China, Chengdu, \\ Sichuan 61173, China \\ ${ }^{2}$ Department of Mathematics, North University of China, Taiyuan, Shanxi 030051, China \\ ${ }^{3}$ Department of Mathematics and Statistics, Georgia State University, Atlanta, GA 30302-4110, USA
}

Correspondence should be addressed to Ling Zhang, lvjinliang415@163.com

Received 1 March 2012; Accepted 1 December 2012

Academic Editor: Nicola Guglielmi

Copyright (C) 2012 Ling Zhang et al. This is an open access article distributed under the Creative Commons Attribution License, which permits unrestricted use, distribution, and reproduction in any medium, provided the original work is properly cited.

The base set of primitive zero-symmetric sign pattern matrices with zero diagonal is $\{1,2, \ldots, 2 n-$ $1\}$. In this paper, the primitive zero-symmetric sign pattern matrices with zero diagonal attaining the maximal base $2 n-1$ are characterized.

\section{Introduction}

A sign pattern matrix (or sign pattern) $A$ is a matrix whose entries are from the set $\{1,-1,0\}$. Notice that for a square sign pattern matrix $A$, in the computation of (the signs of) the entries of the power $A^{k}$, an ambiguous sign may arise when a positive sign is added to a negative sign. So a new symbol \# was introduced in [1] to denote such an ambiguous sign. The powers of a square sign pattern have been investigated to some extent, see, for example, [1-12]. In [1], the set $\Gamma=\{1,-1,0, \#\}$ is defined as the generalized sign set and the matrices with entries in the set $\Gamma$ are called generalized sign pattern matrices, and the addition and multiplication involving the symbol \# are defined as follows:

$$
\begin{aligned}
& (-1)+1=1+(-1)=\# ; \quad a+\#=\# \quad(\forall a \in \Gamma), \\
& 0 \cdot \#=\# \cdot=0 ; \quad b \cdot \#=\# \cdot b=\# \quad(\forall b \in \Gamma \backslash\{0\}) .
\end{aligned}
$$


From now on, we assume that all the matrix operations considered in this paper are operations of the matrices over the set $\Gamma$.

Definition 1.1. A square generalized sign pattern matrix $A$ is called powerful if each power of $A$ contains no \# entry.

In [1], Li et al. introduced the concepts of base and period for (powerful) sign pattern matrices which are the generalizations of the concepts of "index of convergence" and period for square nonnegative matrices. These concepts are extended from (powerful) sign pattern matrices to (square) generalized sign pattern matrices by You et al. in [12] as follows.

Definition 1.2 (see [12]). Let $A$ be a square generalized sign pattern matrix of order $n$ and $A, A^{2}, A^{3}, \ldots$ the sequence of powers of $A$. (Since there are only $4^{n^{2}}$ different generalized sign patterns of order $n$, there must be repetitions in the sequence.) Suppose $A^{l}$ is the first power that is repeated in the sequence, that is, $l$ is the least positive integer such that $A^{l}=A^{l+p}$ holds for some positive integer $p$. Then $l$ is called the generalized base (or simply base) of $A$, and is denoted by $l(A)$. The least positive integer $p$ such that $A^{l}=A^{l+p}$ holds for $l=l(A)$ is called the generalized period (or simply period) of $A$ and is denoted by $p(A)$.

For a sign pattern matrix $A$, we use $|A|$ to denote the $(0,1)$ matrix obtained from $A$ by replacing each nonzero entry by 1 .

A nonnegative square matrix $A$ is primitive if some power $A^{k}>0$. The least such as $k$ is called the primitive exponent (or simply exponent) of $A$, denoted by $\exp (A)$. For convenience, a square sign pattern matrix $A$ is called primitive if $|A|$ is primitive, and in this case we define $\exp (A)=\exp (|A|)$.

Definition 1.3 (see [3]). A square sign pattern matrix $A=\left(a_{i j}\right)_{n \times n}$ is called zero-pattern symmetric (abbreviated zero-symmetric, or simply ZS) if $|A|$ is symmetric.

It is well known that graph-theoretical methods are often useful in the study of the powers of square matrices, so we now introduce some graph-theoretical concepts.

Let $D$ be a digraph with vertex set $V$ and arc set $E$ (which permits loops but no multiple arcs). By assigning a sign of 1 or -1 to each arc of the digraph $D$, we obtain a signed digraph $S$. By a walk $W$ in the digraph $D$ (or the signed digraph $S$ ), we mean a sequence of vertices $\left(v_{0}, v_{1}, v_{2}, \ldots, v_{k}\right)$ such that $e_{i}=\left(v_{i-1}, v_{i}\right)$ is an arc of $D$ for $i=1, \ldots, k$. The number $k$ is called the length of the walk $W$, denoted by $l(W)$. If the vertices $v_{0}, v_{1}, \ldots, v_{k-1}$ are distinct, the walk $W$ is called a path, if $v_{k}=v_{0}$, the path $W$ is called a cycle in $D$ (and in $S$ ). The sign of the walk $W$ in $S$, denoted by $\operatorname{sgn}(W)$, is defined to be $\prod_{i=1}^{k} \operatorname{sgn}\left(e_{i}\right)$ where $\operatorname{sgn}\left(e_{i}\right)$ is the sign of the $\operatorname{arc} e_{i}$.

Let $A=\left(a_{i j}\right)$ be a sign pattern matrix of order $n$. Then the associated digraph $D(A)$ of $A$ is defined to be the digraph with vertex set $V=\{1,2, \ldots, n\}$ and arc set $E=\left\{\left(v_{i}, v_{j}\right)\right.$ | $\left.a_{i j} \neq 0\right\}$. The associated signed digraph $S(A)$ is obtained from $D(A)$ by assigning the sign of $a_{i j}$ to each $\operatorname{arc}\left(v_{i}, v_{j}\right)$ in $D(A)$.

Definition 1.4 (see [10]). Let $D$ be a digraph (permitting loops but no multiple arcs). Digraph $D$ is called primitive if there is a positive integer $k$ such that for all ordered pairs of vertices $v_{i}$ and $v_{j}$ (not necessarily distinct) in $D$, there exists a walk of length $k$ from $v_{i}$ to $v_{j}$. The least such $k$ is called the primitive exponent of $D$, denoted by $\exp (D)$.

It is well known that a digraph $D$ is primitive if and only if $D$ is strongly connected and the greatest common divisor (gcd for short) of the lengths of all the cycles of $D$ is 1 (see [13]). 


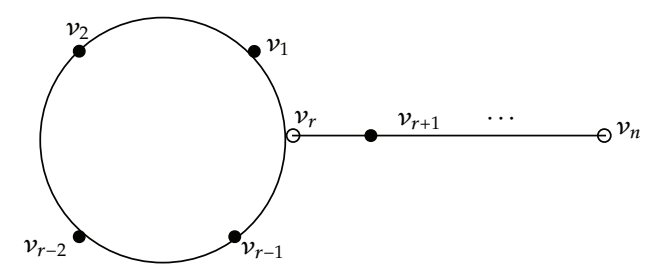

Figure 1: The graph G. (All 2-cycles in $G$ are negative, $r$ is odd, $3 \leq r \leq n$ ).

Definition 1.5 (see [3]). Let $S$ be a signed digraph of order $n$. Then there is a sign pattern matrix $A$ of order $n$ whose associated signed digraph $S(A)$ is $S$. We say that $S$ is powerful if $A$ is powerful. Also we define $l(S)=l(A)$.

We say that a sign pattern matrix $A=\left(a_{i j}\right)$ has zero diagonal if $a_{i i}=0$ for all $i$. A digraph $D$ with vertex set $\left\{v_{1}, \ldots, v_{n}\right\}$ and arc set $E$ is called symmetric provided that $\left(v_{i}, v_{j}\right) \in$ $E$ iff $\left(v_{j}, v_{i}\right) \in E$ for all $i, j$. It is clear that a sign pattern matrix $A$ is ZS iff its associated digraph $D(A)$ is symmetric. For simplicity, we represent a symmetric (signed) digraph by its underlying graph.

The base set of primitive ZS sign pattern matrices and the base set of primitive ZS sign pattern matrices with zero diagonal are given, respectively, in [3, 10]. In [2], Cheng and Liu characterized the primitive ZS sign pattern matrices with the maximum base.

In this paper, we characterize the primitive sign pattern matrices with zero diagonal attaining the maximum base. Our main result is given in the following theorem.

Theorem 1.6. Let $A=\left(a_{i j}\right)$ be an $n \times n$ primitive zero-symmetric sign pattern matrix with zero diagonal. Then

$$
l(A) \leq 2 n-1
$$

and the equality holds if and only if $A$ is nonpowerful and skew symmetric, namely, $a_{i j}=-a_{j i}$ for all $1 \leq i \leq j \leq n$, and the associated digraph $D(A)$ is isomorphic to $G$ (see Figure 1).

The proof of Theorem 1.6 will be given in Section 3 .

\section{Preliminary Results}

In this section, we introduce some theorems, definitions, and lemmas which we need to use in the proof of our main result in Section 3.

In [1], Li et al. showed that if an irreducible sign pattern matrix $A$ is powerful, then $l(A)=l(|A|)$. That is to say the study of the base for a primitive powerful sign pattern matrix is essentially the study of the base (i.e., exponent) for primitive $(0,1)$ matrices. Therefore, for a primitive powerful ZS sign pattern matrix with zero diagonal, Theorem 2.1 gives the base.

Theorem 2.1 (see [8]). Let $A$ be an $n \times n$ primitive symmetric $(0,1)$ matrix with zero diagonal. Then $\exp (A) \leq 2 n-4$ and the primitive exponent set of $n \times n$ primitive symmetric $(0,1)$ matrix with zero diagonal is $\{2,3, \ldots, 2 n-4\} \backslash D$, where $D$ is the set of odd numbers in $\{n-2, n-1, \ldots, 2 n-5\}$. 
Theorem 2.2 (see [10]). Let $A$ be an $n \times n$ primitive ZS sign pattern matrix with zero diagonal. Then $l(A) \leq 2 n-1$.

By Theorems 2.1 and 2.2, the sign pattern matrices with zero diagonal attaining this upper bound $l(A)=2 n-1$ must be nonpowerful. So it remains to consider nonpowerful sign pattern matrices with zero diagonal.

Definition 2.3. Two walks $W_{1}$ and $W_{2}$ in a signed digraph are called a pair of SSSD walks, if they have the same initial vertex, same terminal vertex, and same length, but they have different signs.

Lemma 2.4 (see [8]). Let $D$ be a symmetric digraph. Then $D$ is primitive if and only if $D$ is strongly connected and there exists an odd cycle in $D$.

Lemma 2.5 (see $[1,12]$ ). If $S$ is a primitive signed digraph, then $S$ is nonpowerful if and only if $S$ contains a pair of cycles $C_{1}$ and $C_{2}$, with lengths $p_{1}$ and $p_{2}$, respectively, satisfying one of the following conditions:

$\left(A_{1}\right) p_{1}$ is odd and $p_{2}$ is even and $\operatorname{sgn}\left(C_{2}\right)=-1$;

$\left(A_{2}\right)$ both $p_{1}$ and $p_{2}$ are odd and $\operatorname{sgn}\left(C_{1}\right)=-\operatorname{sgn}\left(C_{2}\right)$.

Lemma 2.6 (see [12]). Let S be a primitive, nonpowerful signed digraph. Then we have the following.

(1) There is an integer $k$ such that there exists a pair of SSSD walks of length $k$ from each vertex $x$ to each vertex $y$ in $S$.

(2) If there exists a pair of SSSD walks of length $k$ from each vertex $x$ to each vertex $y$, then there also exists a pair of SSSD walks of length $k+1$ from each vertex $u$ to each vertex $v$ in $S$.

(3) The minimal such $k$ (as in (1)) is just $l(S)$, the base of $S$.

Lemma 2.7 (see [7]). Suppose that an $n \times n$ sign pattern matrix $A=\left(a_{i j}\right)$ is skew symmetric. Let $S(A)$ be the associated signed digraph of $A$. Let $r$ be an odd integer with $3 \leq r \leq n(n \geq 3)$. Then $l(S(A))=2 n-1$ if and only if $S(A)$ is isomorphic to $G$ (see Figure 1 ).

\section{Main Results}

For an undirected walk $W$ of graph $G$ and two vertices $x, y$ on $W$, we denote by $Q_{W}(x \rightarrow y)$ a shortest path from $x$ to $y$ on $W$ and by $Q(x \rightarrow y)$ a shortest path from $x$ to $y$ on G. For a cycle $C$ of $G$, if $x$ and $y$ are two (not necessarily distinct) vertices on $C$ and $P$ is a path from $x$ to $y$ along $C$, then $C \backslash P$ denotes the path or cycle from $x$ to $y$ along $C$ obtained by deleting the edges of $P$.

Lemma 3.1. Let $A$ be an $n \times n$ primitive nonpowerful ZS sign pattern matrix with zero diagonal. If all the 2 cycles in $S(A)$ are positive, then $l(A) \leq 2 n-2$.

Proof. Since $A$ is primitive, it follows from Lemma 2.4 that $S(A)$ is strongly connected and there is an odd cycle $C^{\prime}$ in $S(A)$ such that $l\left(C^{\prime}\right)=l^{\prime}$. Since $A$ has zero diagonal, there are no loops in $S(A)$ and so $l^{\prime} \geq 3$. Without loss of generality, we assume that $C^{\prime}$ is an odd cycle with the least length in $S(A)$. 
Case 1. There exists at least one negative even cycle in $S(A)$.

Let $C$ be a negative even cycle in $S(A)$. Without loss of generality, we assume that $C$ is a negative even cycle with the least length in $S(A)$. Since all 2 cycles in $S(A)$ are positive, $l(C)=l \geq 4$.

Subcase 1.1. $C$ and $C^{\prime}$ have no common vertices.

Let $P$ be the shortest path from $C$ to $C^{\prime}$. Suppose $P$ intersects $C$ at vertex $u$ and intersects $C^{\prime}$ at vertex $v$ and there are $k$ vertices on $P$ where $k \geq 2$. Let $G_{0}=C \cup P \cup C^{\prime}$. Let $x$ and $y$ be any two (not necessarily distinct) vertices in $S(A)$. Suppose that $P_{1}$ is the shortest path from $x$ to $G_{0}$ and intersects $G_{0}$ at vertex $x^{\prime}$ and $P_{2}$ is the shortest path from $y$ to $G_{0}$ and intersects $G_{0}$ at vertex $y^{\prime}$. Then $0 \leq l\left(P_{1}\right), l\left(P_{2}\right) \leq n-l-l^{\prime}-k+2$, where $l \geq 4$ and $l^{\prime} \geq 3$. By the proof of Case 1 of Lemma 4.5 in [3], there exists a pair of SSSD walks from $x$ to $y$ of length $2 n-2$. Therefore, by Lemma $2.6, l(A) \leq 2 n-2$.

Subcase 1.2. $C$ and $C^{\prime}$ have at least one common vertex.

Let $G_{1}=C \cup C^{\prime}$. Let $x$ and $y$ be any two (not necessarily distinct) vertices in $S(A)$. Suppose that $P_{1}$ is the shortest path from $x$ to $G_{1}$ and intersects $G_{1}$ at vertex $x^{\prime}$ and $P_{2}$ is the shortest path from $y$ to $G_{1}$ and intersects $G_{1}$ at vertex $y^{\prime}$. Denote $C \cap C^{\prime}$ by $R$. Assume $R$ has $m$ vertices, where $1 \leq m \leq \min \left(l, l^{\prime}\right)$, then

$$
0 \leq l\left(P_{1}\right), \quad l\left(P_{2}\right) \leq n-l-l^{\prime}+m .
$$

If $m \geq 2$, then $R$ is a path with vertex set $V(R)=\left\{v_{1}, v_{2}, \ldots, v_{m}\right\}$ and edge set $E(R)=$ $\left\{\left(v_{1}, v_{2}\right),\left(v_{2}, v_{3}\right), \ldots,\left(v_{m-1}, v_{m}\right)\right\}$. In this case, if $m=\min \left(l, l^{\prime}\right)=l$, then there exists another odd cycle with length $l^{\prime}-l+2<l^{\prime}$, which contradicts our assumption that $C^{\prime}$ is an odd cycle with the least length in $S(A)$. Therefore, $m<l$ and if $m=\min \left(l, l^{\prime}\right)$, then $m=l^{\prime}$.

Subcase 1.2.1. $x^{\prime}=y^{\prime}$ and $1 \leq m \leq \min \left(l, l^{\prime}\right)$.

Subcase 1.2.1.1. $x^{\prime}=y^{\prime} \in C \backslash R$. See Figure 2(a).

We consider two subcases: the subcase $1 \leq m<\min \left(l, l^{\prime}\right)$ and the subcase $m=$ $\min \left(l, l^{\prime}\right)=l^{\prime}$.

First, we consider the subcase $1 \leq m<\min \left(l, l^{\prime}\right)$. Without loss of generality, we assume that $l\left(Q_{C \backslash R}\left(x^{\prime} \rightarrow v_{1}\right)\right) \leq l\left(Q_{C \backslash R}\left(x^{\prime} \rightarrow v_{m}\right)\right)$. Let $w=l\left(P_{1}\right)+l(C)+l\left(P_{2}\right)$. If $w$ is even, set

$$
W^{\prime}=P_{1}+C+P_{2}
$$

Otherwise, set

$$
W^{\prime \prime}=P_{1}+Q_{C \backslash R}\left(x^{\prime} \longrightarrow v_{1}\right)+Q_{C^{\prime} \backslash R}\left(v_{1} \longrightarrow v_{m}\right)+Q_{C \backslash R}\left(v_{m} \longrightarrow y^{\prime}\right)+P_{2} .
$$

Let

$$
W_{1}=\left\{\begin{array}{ll}
W^{\prime}+C, & w \text { is even, } \\
W^{\prime \prime}+C, & \text { otherwise }
\end{array} \quad W_{2}= \begin{cases}W^{\prime}+\frac{l}{2} C_{2}, & w \text { is even } \\
W^{\prime \prime}+\frac{l}{2} C_{2}, & \text { otherwise }\end{cases}\right.
$$


where $C_{2}$ is a positive 2-cycle that contains vertex $x$. Since $C^{\prime}$ is odd, $l\left(Q_{C^{\prime} \backslash R}\left(v_{1} \rightarrow v_{m}\right)\right)$ and $l\left(Q_{R}\left(v_{1} \rightarrow v_{m}\right)\right)$ have different parity. Therefore, both $l\left(W_{1}\right)$ and $l\left(W_{2}\right)$ are even. Then, if $w$ is even,

$$
l\left(W_{1}\right)=l\left(W_{2}\right) \leq 2\left(n-l-l^{\prime}+m\right)+2 l=2 n-2 l^{\prime}+2 m .
$$

Otherwise,

$$
l\left(W_{1}\right)=l\left(W_{2}\right) \leq 2\left(n-l-l^{\prime}+m\right)+(l-m+1)+\left(l^{\prime}-m+1\right)+l=2 n-l^{\prime}+2 .
$$

Since both $l\left(W_{1}\right)$ and $l\left(W_{2}\right)$ are even, it follows that $l\left(W_{1}\right)=l\left(W_{2}\right) \leq 2 n-2$. We see that the pair $W_{1}, W_{2}$ is a pair of SSSD walks with even length. Therefore, there exists a pair of SSSD walks from $x$ to $y$ with length $2 n-2$.

Then, we consider the subcase $m=\min \left(l, l^{\prime}\right)=l^{\prime}$. Without loss of generality, we assume that $l\left(Q_{C \backslash R}\left(x^{\prime} \rightarrow v_{1}\right)\right) \leq l\left(Q_{C \backslash R}\left(x^{\prime} \rightarrow v_{m}\right)\right)$. Let

$$
w=l\left(P_{1}\right)+2 l\left(Q_{C \backslash R}\left(x^{\prime} \longrightarrow v_{1}\right)\right)+l\left(P_{2}\right)
$$

If $w$ is even, set

$$
W^{\prime}=P_{1}+Q_{C \backslash R}\left(x^{\prime} \longrightarrow v_{1}\right)+Q_{C \backslash R}\left(v_{1} \longrightarrow x^{\prime}\right)+P_{2}
$$

Otherwise, set

$$
W^{\prime \prime}=P_{1}+Q_{C \backslash R}\left(x^{\prime} \longrightarrow v_{1}\right)+Q_{C^{\prime} \backslash R}\left(v_{1} \longrightarrow v_{m}\right)+Q_{C \backslash R}\left(v_{m} \longrightarrow x^{\prime}\right)+P_{2} .
$$

Let

$$
W_{1}=\left\{\begin{array}{ll}
W^{\prime}+C, & w \text { is even, } \\
W^{\prime \prime}+C, & \text { otherwise }
\end{array} \quad W_{2}=\left\{\begin{array}{l}
W^{\prime}+\frac{l}{2} C_{2}, \quad w \text { is even } \\
W^{\prime \prime}+\frac{l}{2} C_{2}, \quad \text { otherwise }
\end{array}\right.\right.
$$

where $C_{2}$ is a positive 2-cycle that contains vertex $x$. Since $Q_{C \backslash R}\left(x^{\prime} \rightarrow v_{1}\right)+Q_{C^{\prime} \backslash R}\left(v_{1} \rightarrow v_{m}\right)+$ $Q_{C \backslash R}\left(v_{m} \rightarrow x^{\prime}\right)$ is an odd cycle, $l\left(Q_{C \backslash R}\left(x^{\prime} \rightarrow v_{1}\right)\right)$ and $l\left(Q_{C^{\prime} \backslash R}\left(v_{1} \rightarrow v_{m}\right)+Q_{C \backslash R}\left(v_{m} \rightarrow x^{\prime}\right)\right)$ have different parity. Therefore, both $l\left(W_{1}\right)$ and $l\left(W_{2}\right)$ are even. Then, if $w$ is even,

$$
l\left(W_{1}\right)=l\left(W_{2}\right) \leq 2(n-l)+\left(l-l^{\prime}+1\right)+l=2 n-l^{\prime}+1 .
$$

Otherwise,

$$
l\left(W_{1}\right)=l\left(W_{2}\right) \leq 2(n-l)+\left(l-l^{\prime}+2\right)+l=2 n-l^{\prime}+2 .
$$




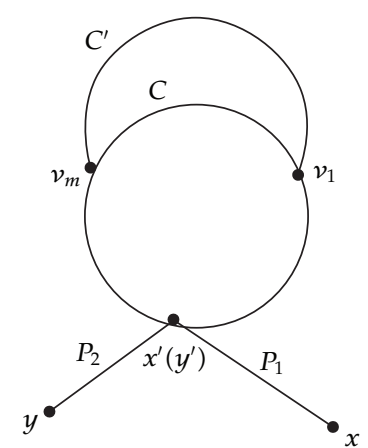

(a)

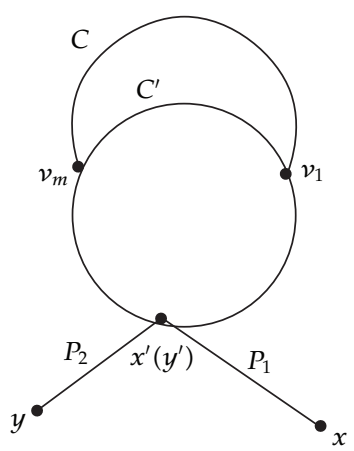

(b)

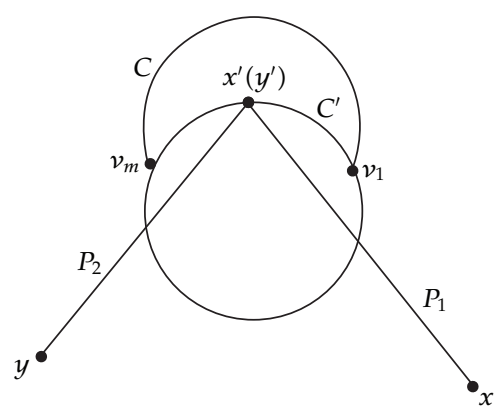

(c)

Figure 2: Illustrate for Subcase 1.2.1 of Lemma 3.1.

Since both $l\left(W_{1}\right)$ and $l\left(W_{2}\right)$ are even, it follows that $l\left(W_{1}\right)=l\left(W_{2}\right) \leq 2 n-2$. We see that the pair $W_{1}, W_{2}$ is a pair of SSSD walks with even length. Therefore, there exists a pair of SSSD walks from $x$ to $y$ with length $2 n-2$.

Subcase 1.2.1.2. $x^{\prime}=y^{\prime} \in C^{\prime} \backslash R$. See Figure 2(b).

The proof for this subcase is similar to that of the Subcase 1.2.1.1 and is omitted.

Subcase 1.2.1.3. $x^{\prime}=y^{\prime} \in R$. See Figure 2(c).

Let $w=l\left(P_{1}\right)+l\left(P_{2}\right)$. If $w$ is even, set

$$
W^{\prime}=P_{1}+P_{2}
$$

Otherwise, set

$$
W^{\prime \prime}=P_{1}+P_{2}+C^{\prime}
$$

Let

$$
W_{1}=\left\{\begin{array}{ll}
W^{\prime}+C, & w \text { is even, } \\
W^{\prime \prime}+C, & \text { otherwise }
\end{array} \quad W_{2}=\left\{\begin{array}{l}
W^{\prime}+\frac{l}{2} C_{2}, \quad w \text { is even } \\
W^{\prime \prime}+\frac{l}{2} C_{2}, \quad \text { otherwise }
\end{array}\right.\right.
$$

where $C_{2}$ is a positive 2-cycle that contains vertex $x$. Since $l^{\prime}$ is odd, we see that both $l\left(W_{1}\right)$ and $l\left(W_{2}\right)$ are even. Then, if $w$ is even,

$$
l\left(W_{1}\right)=l\left(W_{2}\right) \leq 2\left(n-l-l^{\prime}+m\right)+l=2 n-l-2 l^{\prime}+2 m .
$$

Otherwise,

$$
l\left(W_{1}\right)=l\left(W_{2}\right) \leq 2\left(n-l-l^{\prime}+m\right)+l^{\prime}+l=2 n-l-l^{\prime}+2 m .
$$




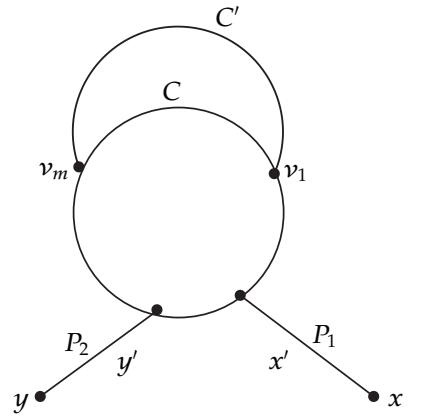

(a)

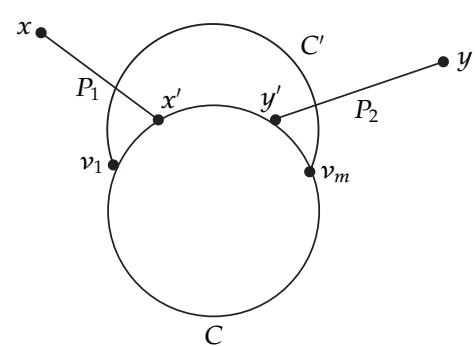

(d)

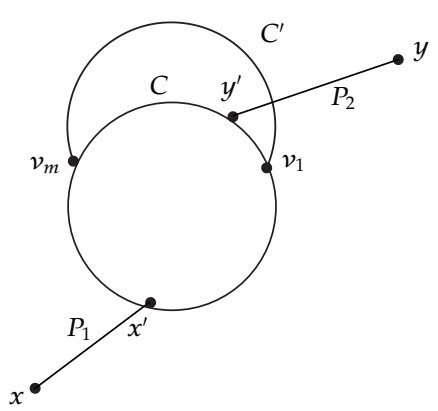

(b)

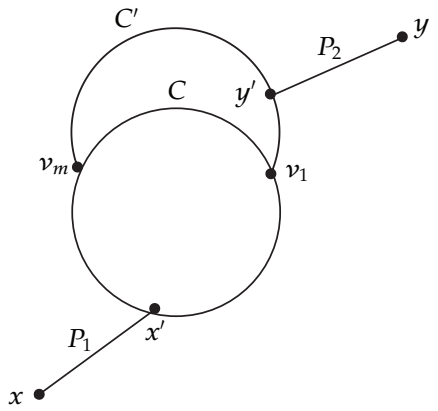

(c)

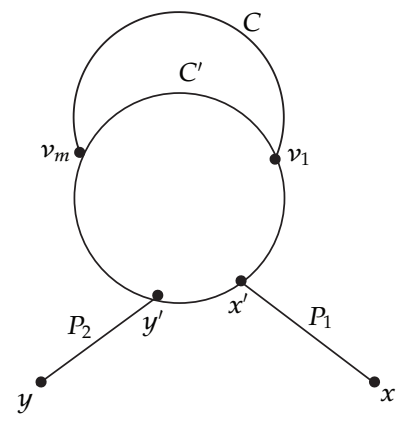

(f)

Figure 3: Illustration for Subcases 1.2.2 and 2.2 of Lemma 3.1.

Since both $l\left(W_{1}\right)$ and $l\left(W_{2}\right)$ are even, it follows that $l\left(W_{1}\right)=l\left(W_{2}\right) \leq 2 n-2$. We see that the pair $W_{1}, W_{2}$ is a pair of SSSD walks with even length. Therefore, there exists a pair of SSSD walks from $x$ to $y$ with length $2 n-2$.

Subcase 1.2.2. $x^{\prime} \neq y^{\prime}$ and $2 \leq m \leq \min \left(l, l^{\prime}\right)$.

Subcase 1.2.2.1. $x^{\prime} \in C \backslash R$ and $y^{\prime} \in C \backslash R$. See Figure 3(a).

Without loss of generality, we assume that $l\left(Q_{C \backslash R}\left(x^{\prime} \rightarrow v_{1}\right)\right) \leq l\left(Q_{C \backslash R}\left(y^{\prime} \rightarrow v_{1}\right)\right)$. Let $w=l\left(P_{1}\right)+l\left(Q_{C \backslash R}\left(x^{\prime} \rightarrow v_{1}\right)\right)+l\left(Q_{R}\left(v_{1} \rightarrow v_{m}\right)\right)+l\left(Q_{C \backslash R}\left(v_{m} \rightarrow y^{\prime}\right)\right)+l\left(P_{2}\right)$. If $w$ is even, set

$$
W^{\prime}=P_{1}+Q_{C \backslash R}\left(x^{\prime} \longrightarrow v_{1}\right)+Q_{R}\left(v_{1} \longrightarrow v_{m}\right)+Q_{C \backslash R}\left(v_{m} \longrightarrow y^{\prime}\right)+P_{2}
$$

Otherwise, set

$$
W^{\prime \prime}=P_{1}+Q_{C \backslash R}\left(x^{\prime} \longrightarrow v_{1}\right)+Q_{C^{\prime} \backslash R}\left(v_{1} \longrightarrow v_{m}\right)+Q_{C \backslash R}\left(v_{m} \longrightarrow y^{\prime}\right)+P_{2} .
$$

Let

$$
W_{1}=\left\{\begin{array}{ll}
W^{\prime}+C, & w \text { is even, } \\
W^{\prime \prime}+C, & \text { otherwise, }
\end{array} \quad W_{2}= \begin{cases}W^{\prime}+\frac{l}{2} C_{2}, & w \text { is even, } \\
W^{\prime \prime}+\frac{l}{2} C_{2}, & \text { otherwise }\end{cases}\right.
$$

where $C_{2}$ is a positive 2-cycle that contains vertex $x$. Since $C^{\prime}$ is odd, $l\left(Q_{C^{\prime} \backslash R}\left(v_{1} \rightarrow v_{m}\right)\right)$ and $l\left(Q_{R}\left(v_{1} \rightarrow v_{m}\right)\right)$ have different parity. Therefore, both $l\left(W_{1}\right)$ and $l\left(W_{2}\right)$ are even. Noting that 
$W_{3}=P_{1}+Q_{C \backslash R}\left(x^{\prime} \rightarrow v_{1}\right)+Q_{R}\left(v_{1} \rightarrow v_{m}\right)+Q_{C \backslash R}\left(v_{m} \rightarrow y^{\prime}\right)$ and $W_{4}=P_{1}+Q_{C \backslash R}\left(x^{\prime} \rightarrow\right.$ $\left.v_{1}\right)+Q_{C^{\prime} \backslash R}\left(v_{1} \rightarrow v_{m}\right)+Q_{C \backslash R}\left(v_{m} \rightarrow y^{\prime}\right)$ are paths of $S(A)$, we have $l\left(W_{3}\right), l\left(W_{4}\right) \leq n-1$. Then

$$
l\left(W_{1}\right)=l\left(W_{2}\right) \leq(n-1)+\left(n-l-l^{\prime}+m\right)+l=2 n-l^{\prime}+m-1 .
$$

Since both $l\left(W_{1}\right)$ and $l\left(W_{2}\right)$ are even, it follows that $l\left(W_{1}\right)=l\left(W_{2}\right) \leq 2 n-2$. We see that the pair $W_{1}, W_{2}$ is a pair of SSSD walks with even length. Therefore, there exists a pair of SSSD walks from $x$ to $y$ with length $2 n-2$.

Subcase 1.2.2.2. $x^{\prime} \in C \backslash R$ and $y^{\prime} \in R$. See Figure 3(b).

Without loss of generality, we assume that $l\left(Q_{C \backslash R}\left(x^{\prime} \rightarrow v_{1}\right)\right) \leq l\left(Q_{C \backslash R}\left(x^{\prime} \rightarrow v_{m}\right)\right)$. Then we consider two subcases: the subcase $x^{\prime} \in C \backslash R, y^{\prime} \in R$ and $y^{\prime} \neq v_{1}$ and the subcase $x^{\prime} \in C \backslash R$ and $y^{\prime}=v_{1}$.

First, we consider the subcase $x^{\prime} \in C \backslash R, y^{\prime} \in R$ and $y^{\prime} \neq v_{1}$. Let $w=l\left(P_{1}\right)+l\left(Q_{C \backslash R}\left(x^{\prime} \rightarrow\right.\right.$ $\left.\left.v_{1}\right)\right)+l\left(Q_{R}\left(v_{1} \rightarrow y^{\prime}\right)\right)+l\left(P_{2}\right)$. If $w$ is even, set

$$
W^{\prime}=P_{1}+Q_{C \backslash R}\left(x^{\prime} \longrightarrow v_{1}\right)+Q_{R}\left(v_{1} \longrightarrow y^{\prime}\right)+P_{2}
$$

Otherwise, set

$$
W^{\prime \prime}=P_{1}+Q_{C \backslash R}\left(x^{\prime} \longrightarrow v_{1}\right)+Q_{C^{\prime} \backslash R}\left(v_{1} \longrightarrow v_{m}\right)+Q_{R}\left(v_{m} \longrightarrow y^{\prime}\right)+P_{2}
$$

Let

$$
W_{1}=\left\{\begin{array}{ll}
W^{\prime}+C, & w \text { is even, } \\
W^{\prime \prime}+C, & \text { otherwise }
\end{array} \quad W_{2}= \begin{cases}W^{\prime}+\frac{l}{2} C_{2}, & w \text { is even } \\
W^{\prime \prime}+\frac{l}{2} C_{2}, & \text { otherwise }\end{cases}\right.
$$

where $C_{2}$ is a positive 2-cycle that contains vertex $x$. Since $C^{\prime}$ is odd, $l\left(Q_{R}\left(v_{1} \rightarrow y^{\prime}\right)\right)$ and $l\left(Q_{C^{\prime} \backslash R}\left(v_{1} \rightarrow v_{m}\right)\right)+l\left(Q_{R}\left(v_{m} \rightarrow y^{\prime}\right)\right)$ have different parity. Therefore, both $l\left(W_{1}\right)$ and $l\left(W_{2}\right)$ are even. Noting that $W_{3}=P_{1}+Q_{C \backslash R}\left(x^{\prime} \rightarrow v_{1}\right)+Q_{R}\left(v_{1} \rightarrow y^{\prime}\right)$ and $W_{4}=P_{1}+Q_{C \backslash R}\left(x^{\prime} \rightarrow\right.$ $\left.v_{1}\right)+Q_{C^{\prime} \backslash R}\left(v_{1} \rightarrow v_{m}\right)+Q_{R}\left(v_{m} \rightarrow y^{\prime}\right)$ are two paths of $S(A)$, then we have $l\left(W_{3}\right), l\left(W_{4}\right) \leq n-1$. Then

$$
l\left(W_{1}\right)=l\left(W_{2}\right) \leq(n-1)+\left(n-l-l^{\prime}+m\right)+l=2 n-l^{\prime}+m-1 .
$$

Since both $l\left(W_{1}\right)$ and $l\left(W_{2}\right)$ are even, it follows that $l\left(W_{1}\right)=l\left(W_{2}\right) \leq 2 n-2$. We see that the pair $W_{1}, W_{2}$ is a pair of SSSD walks with even length. Therefore, there exists a pair of SSSD walks from $x$ to $y$ with length $2 n-2$.

Then, we consider the subcase $x^{\prime} \in C \backslash R$ and $y^{\prime}=v_{1}$. Let $w=l\left(P_{1}\right)+l\left(Q_{C \backslash R}\left(x^{\prime} \rightarrow\right.\right.$ $\left.\left.v_{m}\right)\right)+l\left(Q_{R}\left(v_{m} \rightarrow v_{1}\left(y^{\prime}\right)\right)\right)+l\left(P_{2}\right)$. If $w$ is even, set

$$
W^{\prime}=P_{1}+Q_{C \backslash R}\left(x^{\prime} \longrightarrow v_{m}\right)+Q_{R}\left(v_{m} \longrightarrow v_{1}\left(y^{\prime}\right)\right)+P_{2}
$$


Otherwise, set

$$
W^{\prime \prime}=P_{1}+Q_{C \backslash R}\left(x^{\prime} \longrightarrow v_{m}\right)+Q_{C^{\prime} \backslash R}\left(v_{m} \longrightarrow v_{1}\left(y^{\prime}\right)\right)+P_{2}
$$

Let

$$
W_{1}=\left\{\begin{array}{ll}
W^{\prime}+C, & w \text { is even, } \\
W^{\prime \prime}+C, & \text { otherwise }
\end{array} \quad W_{2}= \begin{cases}W^{\prime}+\frac{l}{2} C_{2}, & w \text { is even } \\
W^{\prime \prime}+\frac{l}{2} C_{2}, & \text { otherwise }\end{cases}\right.
$$

where $C_{2}$ is a positive 2-cycle that contains vertex $x$. Since $C^{\prime}$ is odd, $l\left(Q_{R}\left(v_{m} \rightarrow v_{1}\left(y^{\prime}\right)\right)\right)$ and $l\left(Q_{C^{\prime} \backslash R}\left(v_{m} \rightarrow v_{1}\left(y^{\prime}\right)\right)\right)$ have different parity. Therefore, both $l\left(W_{1}\right)$ and $l\left(W_{2}\right)$ are even. Noting that $W_{3}=P_{1}+Q_{C \backslash R}\left(x^{\prime} \rightarrow v_{m}\right)+Q_{R}\left(v_{m} \rightarrow v_{1}\left(y^{\prime}\right)\right)$ and $W_{4}=P_{1}+Q_{C \backslash R}\left(x^{\prime} \rightarrow\right.$ $\left.v_{m}\right)+Q_{C^{\prime} \backslash R}\left(v_{m} \rightarrow v_{1}\left(y^{\prime}\right)\right)$ are two paths of $S(A)$, we have $l\left(W_{3}\right), l\left(W_{4}\right) \leq n-1$. Then

$$
l\left(W_{1}\right)=l\left(W_{2}\right) \leq(n-1)+\left(n-l-l^{\prime}+m\right)+l=2 n-l^{\prime}+m-1 .
$$

Since both $l\left(W_{1}\right)$ and $l\left(W_{2}\right)$ are even, it follows that $l\left(W_{1}\right)=l\left(W_{2}\right) \leq 2 n-2$. We see that the pair $W_{1}, W_{2}$ is a pair of SSSD walks with even length. Therefore, there exists a pair of SSSD walks from $x$ to $y$ with length $2 n-2$.

Subcase 1.2.2.3. $x^{\prime} \in C \backslash R$ and $y^{\prime} \in C^{\prime} \backslash R$. See Figure 3(c).

The proof for this subcase is similar to that of the Subcase 1.2.2.1 and is omitted.

Subcase 1.2.2.4. $x^{\prime} \in R$ and $y^{\prime} \in R$. See Figure 3(d).

Let $w=l\left(P_{1}\right)+l\left(Q_{C^{\prime}}\left(x^{\prime} \rightarrow y^{\prime}\right)\right)+l\left(P_{2}\right)$. If $w$ is even, set

$$
W^{\prime}=P_{1}+Q_{C^{\prime}}\left(x^{\prime} \longrightarrow y^{\prime}\right)+P_{2} .
$$

Otherwise, set

$$
W^{\prime \prime}=P_{1}+C^{\prime} \backslash Q_{C^{\prime}}\left(x^{\prime} \longrightarrow y^{\prime}\right)+P_{2}
$$

Let

$$
W_{1}=\left\{\begin{array}{ll}
W^{\prime}+C, & w \text { is even, } \\
W^{\prime \prime}+C, & \text { otherwise }
\end{array} \quad W_{2}=\left\{\begin{array}{l}
W^{\prime}+\frac{l}{2} C_{2}, \quad w \text { is even } \\
W^{\prime \prime}+\frac{l}{2} C_{2}, \quad \text { otherwise }
\end{array}\right.\right.
$$


where $C_{2}$ is a positive 2-cycle that contains vertex $x$. Since $C^{\prime}$ is odd, $l\left(Q_{C^{\prime}}\left(x^{\prime} \rightarrow y^{\prime}\right)\right)$ and $l\left(C^{\prime} \backslash Q_{C^{\prime}}\left(x^{\prime} \rightarrow y^{\prime}\right)\right)$ have different parity. Therefore, both $l\left(W_{1}\right)$ and $l\left(W_{2}\right)$ are even. Then if $w$ is even,

$$
l\left(W_{1}\right)=l\left(W_{2}\right) \leq 2\left(n-l-l^{\prime}+m\right)+\frac{l^{\prime}-1}{2}+l=2 n+2 m-l-\frac{3 l^{\prime}}{2}-\frac{1}{2} .
$$

Otherwise,

$$
l\left(W_{1}\right)=l\left(W_{2}\right) \leq 2\left(n-l-l^{\prime}+m\right)+\left(l^{\prime}-1\right)+l=2 n-l-l^{\prime}+2 m-1 .
$$

Since both $l\left(W_{1}\right)$ and $l\left(W_{2}\right)$ are even, it follows that $l\left(W_{1}\right)=l\left(W_{2}\right) \leq 2 n-2$. We see that the pair $W_{1}, W_{2}$ is a pair of SSSD walks with even length. Therefore, there exists a pair of SSSD walks from $x$ to $y$ with length $2 n-2$.

Subcase 1.2.2.5. $x^{\prime} \in R$ and $y^{\prime} \in C^{\prime} \backslash R$. See Figure 3(e).

The proof for this subcase is similar to that of Subcase 1.2.2.4, so we omit it.

Subcase 1.2.2.6. $x^{\prime} \in C^{\prime} \backslash R$ and $y^{\prime} \in C^{\prime} \backslash R$. See Figure 3(f).

Without loss of generality, we assume that $l\left(Q_{C^{\prime} \backslash R}\left(x^{\prime} \rightarrow v_{1}\right)\right) \leq l\left(Q_{C^{\prime} \backslash R}\left(y^{\prime} \rightarrow v_{1}\right)\right)$. Let $w=l\left(P_{1}\right)+l\left(Q_{C^{\prime} \backslash R}\left(x^{\prime} \rightarrow v_{1}\right)\right)+l\left(Q_{R}\left(v_{1} \rightarrow v_{m}\right)\right)+l\left(Q_{C^{\prime} \backslash R}\left(v_{m} \rightarrow y^{\prime}\right)\right)+l\left(P_{2}\right)$. If $w$ is even, set

$$
W^{\prime}=P_{1}+Q_{C^{\prime} \backslash R}\left(x^{\prime} \longrightarrow v_{1}\right)+Q_{R}\left(v_{1} \longrightarrow v_{m}\right)+Q_{C^{\prime} \backslash R}\left(v_{m} \longrightarrow y^{\prime}\right)+P_{2}
$$

Otherwise, set

$$
W^{\prime \prime}=P_{1}+Q_{C^{\prime} \backslash R}\left(x^{\prime} \longrightarrow y^{\prime}\right)+Q_{C^{\prime} \backslash R}\left(y^{\prime} \longrightarrow v_{m}\right)+Q_{C^{\prime} \backslash R}\left(v_{m} \longrightarrow y^{\prime}\right)+P_{2} .
$$

Let

$$
W_{1}=\left\{\begin{array}{ll}
W^{\prime}+C, & w \text { is even, } \\
W^{\prime \prime}+C, & \text { otherwise }
\end{array} \quad W_{2}= \begin{cases}W^{\prime}+\frac{l}{2} C_{2}, & w \text { is even } \\
W^{\prime \prime}+\frac{l}{2} C_{2}, & \text { otherwise }\end{cases}\right.
$$

where $C_{2}$ is a positive 2-cycle that contains vertex $x$. Since $C^{\prime}$ is odd, $l\left(Q_{C^{\prime} \backslash R}\left(x^{\prime} \rightarrow y^{\prime}\right)\right)$ and $l\left(Q_{C^{\prime} \backslash R}\left(x^{\prime} \rightarrow v_{1}\right)\right)+l\left(Q_{R}\left(v_{1} \rightarrow v_{m}\right)\right)+l\left(Q_{C^{\prime} \backslash R}\left(v_{m} \rightarrow y^{\prime}\right)\right)$ have different parity. Therefore, both $l\left(W_{1}\right)$ and $l\left(W_{2}\right)$ are even. Noting that $W_{3}=P_{1}+Q_{C^{\prime} \backslash R}\left(x^{\prime} \rightarrow v_{1}\right)+Q_{R}\left(v_{1} \rightarrow v_{m}\right)+Q_{C^{\prime} \backslash R}\left(v_{m} \rightarrow\right.$ $\left.y^{\prime}\right)$ and $W_{4}=P_{1}+Q_{C^{\prime} \backslash R}\left(x^{\prime} \rightarrow y^{\prime}\right)+Q_{C^{\prime} \backslash R}\left(y^{\prime} \rightarrow v_{m}\right)$ are two paths of $S(A)$, then we have $l\left(W_{3}\right) \leq n-1$ and $l\left(W_{4}\right) \leq n-m$. Then, if $w$ is even,

$$
l\left(W_{1}\right)=l\left(W_{2}\right) \leq(n-1)+\left(n-l-l^{\prime}+m\right)+l=2 n-l^{\prime}+m-1 .
$$

Otherwise,

$$
l\left(W_{1}\right)=l\left(W_{2}\right) \leq\left(n-l-l^{\prime}+m\right)+(n-m)+\left(l^{\prime}-m-1\right)+l=2 n-m-1 .
$$




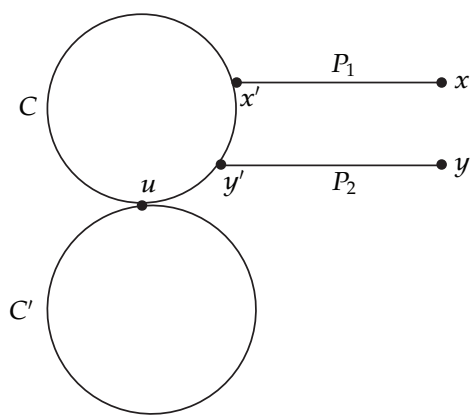

(a)

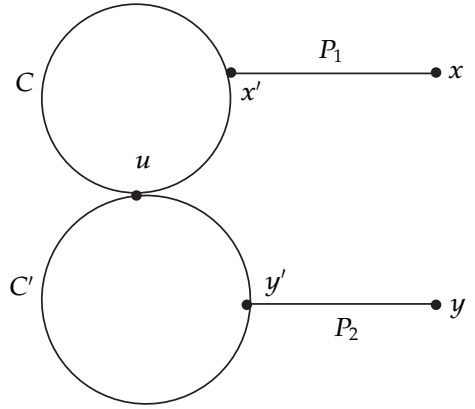

(b)

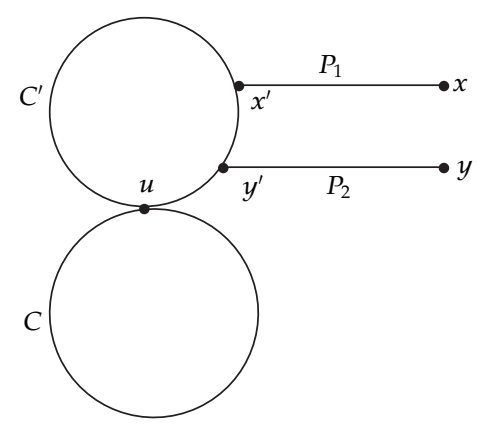

(c)

Figure 4: Illustrate for Subcase 1.2.3 of Lemma 3.1.

Since both $l\left(W_{1}\right)$ and $l\left(W_{2}\right)$ are even, it follows that $l\left(W_{1}\right)=l\left(W_{2}\right) \leq 2 n-2$. We see that the pair $W_{1}, W_{2}$ is a pair of SSSD walks with even length. Therefore, there exists a pair of SSSD walks from $x$ to $y$ with length $2 n-2$.

Subcase 1.2.3. $m=1$ and $x^{\prime} \neq y^{\prime}$. We assume that $V(C) \cap V\left(C^{\prime}\right)=\{u\}$.

Subcase 1.2.3.1. $x^{\prime} \in C$ and $y^{\prime} \in C$. See Figure 4(a).

Let $w=l\left(P_{1}\right)+l\left(Q_{C}\left(x^{\prime} \rightarrow u\right)\right)+l\left(Q_{C}\left(u \rightarrow y^{\prime}\right)\right)+l\left(P_{2}\right)$. If $w$ is even, set

$$
W^{\prime}=P_{1}+Q_{C}\left(x^{\prime} \longrightarrow u\right)+Q_{C}\left(u \longrightarrow y^{\prime}\right)+P_{2} .
$$

Otherwise, set

$$
W^{\prime \prime}=P_{1}+Q_{C}\left(x^{\prime} \longrightarrow u\right)+Q_{C}\left(u \longrightarrow y^{\prime}\right)+P_{2}+C^{\prime}
$$

Let

$$
W_{1}=\left\{\begin{array}{ll}
W^{\prime}+C, & w \text { is even, } \\
W^{\prime \prime}+C, & \text { otherwise }
\end{array} \quad W_{2}=\left\{\begin{array}{l}
W^{\prime}+\frac{l}{2} C_{2}, \quad w \text { is even } \\
W^{\prime \prime}+\frac{l}{2} C_{2}, \quad \text { otherwise }
\end{array}\right.\right.
$$


where $C_{2}$ is a positive 2-cycle that contains vertex $x$. Since $l^{\prime}$ is odd, both $l\left(W_{1}\right)$ and $l\left(W_{2}\right)$ are even. Then, if $w$ is even,

$$
l\left(W_{1}\right)=l\left(W_{2}\right) \leq 2\left(n-l-l^{\prime}+1\right)+(l-1)+l=2 n-2 l^{\prime}+1 .
$$

Otherwise,

$$
l\left(W_{1}\right)=l\left(W_{2}\right) \leq 2\left(n-l-l^{\prime}+1\right)+(l-1)+l^{\prime}+l=2 n-l^{\prime}+1 .
$$

Since both $l\left(W_{1}\right)$ and $l\left(W_{2}\right)$ are even, it follows that $l\left(W_{1}\right)=l\left(W_{2}\right) \leq 2 n-2$. We see that the pair $W_{1}, W_{2}$ is a pair of SSSD walks with even length. Therefore, there exists a pair of SSSD walks from $x$ to $y$ with length $2 n-2$.

Subcase 1.2.3.2. $x^{\prime} \in C, y^{\prime} \in C^{\prime}$, and $x^{\prime} \neq y^{\prime} \neq u$. See Figure $4(\mathrm{~b})$.

Let $w=l\left(P_{1}\right)+l\left(Q_{C}\left(x^{\prime} \rightarrow u\right)\right)+l\left(Q_{C^{\prime}}\left(u \rightarrow y^{\prime}\right)\right)+l\left(P_{2}\right)$. If $w$ is even, set

$$
W^{\prime}=P_{1}+Q_{C}\left(x^{\prime} \longrightarrow u\right)+Q_{C^{\prime}}\left(u \longrightarrow y^{\prime}\right)+P_{2} .
$$

Otherwise, set

$$
W^{\prime \prime}=P_{1}+Q_{C}\left(x^{\prime} \longrightarrow u\right)+C^{\prime} \backslash Q_{C^{\prime}}\left(u \longrightarrow y^{\prime}\right)+P_{2}
$$

Let

$$
W_{1}=\left\{\begin{array}{ll}
W^{\prime}+C, & w \text { is even, } \\
W^{\prime \prime}+C, & \text { otherwise }
\end{array} \quad W_{2}= \begin{cases}W^{\prime}+\frac{l}{2} C_{2}, & w \text { is even } \\
W^{\prime \prime}+\frac{l}{2} C_{2}, & \text { otherwise }\end{cases}\right.
$$

where $C_{2}$ is a positive 2-cycle that contains vertex $x$. Since $C^{\prime}$ is odd, $l\left(C^{\prime} \backslash Q_{C^{\prime}}\left(u \rightarrow y^{\prime}\right)\right)$ and $l\left(Q_{C^{\prime}}\left(u \rightarrow y^{\prime}\right)\right)$ have different parity. Therefore, both $l\left(W_{1}\right)$ and $l\left(W_{2}\right)$ are even. Noting that $W_{3}=P_{1}+Q_{C}\left(x^{\prime} \rightarrow u\right)+Q_{C^{\prime}}\left(u \rightarrow y^{\prime}\right)$ and $W_{4}=P_{1}+Q_{C}\left(x^{\prime} \rightarrow u\right)+C^{\prime} \backslash Q_{C^{\prime}}\left(u \rightarrow y^{\prime}\right)$ are two paths of $S(A)$, we have $l\left(W_{3}\right), l\left(W_{4}\right) \leq n-1$. Then,

$$
l\left(W_{1}\right)=l\left(W_{2}\right) \leq(n-1)+\left(n-l-l^{\prime}+1\right)+l=2 n-l^{\prime} .
$$

Since both $l\left(W_{1}\right)$ and $l\left(W_{2}\right)$ are even, it follows that $l\left(W_{1}\right)=l\left(W_{2}\right) \leq 2 n-2$. We see that the pair $W_{1}, W_{2}$ is a pair of SSSD walks with even length. Therefore, there exists a pair of SSSD walks from $x$ to $y$ with length $2 n-2$.

Subcase 1.2.3.3. $x^{\prime} \in C^{\prime}$ and $y^{\prime} \in C^{\prime}$. See Figure 4(c).

The proof for this subcase is similar to that of the Subcase 1.2.3.1 and is omitted.

Thus in each of the above subcases, there exists a pair of SSSD walks from $x$ to $y$ with length $2 n-2$. Therefore, we get $l(A) \leq 2 n-2$ by Lemma 2.6.

Case 2. There exists no negative even cycle in $S(A)$. 
Since $A$ is primitive, nonpowerful and there exist no negative even cycles in $S(A)$, it follows from Lemma 2.5 that there exist two odd cycles $C$ and $C^{\prime}$ with different signs in $S(A)$. We assume that $l(C)=l$ and $l\left(C^{\prime}\right)=l^{\prime}$. Since the trace of $A$ is zero, there are no loops in $S(A)$ and so $l, l^{\prime} \geq 3$. Without loss of generality, we assume $l \geq l^{\prime} \geq 3$.

Subcase 2.1. $C$ and $C^{\prime}$ have no common vertices.

Let $P$ be the shortest path from $C$ to $C^{\prime}$. Suppose $P$ intersects $C$ at vertex $u$ and intersects $C^{\prime}$ at vertex $v$ and there are $k$ vertices on $P$ where $k \geq 2$. Let $G_{2}=C \cup P \cup C^{\prime}$. Let $x$ and $y$ be two arbitrary (not necessarily distinct) vertices in $S(A)$. Suppose that $P_{1}$ is the shortest path from $x$ to $G_{2}$ and intersects $G_{2}$ at vertex $x^{\prime}$ and $P_{2}$ is the shortest path from $y$ to $G_{2}$ and intersects $G_{2}$ at vertex $y^{\prime}$. Then

$$
0 \leq l\left(P_{1}\right), \quad l\left(P_{2}\right) \leq n-l-l^{\prime}-k+2 .
$$

Since $C$ and $C^{\prime}$ have diffident signs, if there exists an even walk $W$ with length $l(W) \leq 2 n-2-l$, then $W_{1}=W+C$ and $W_{2}=W+\left(\left(l-l^{\prime}\right) / 2\right) C_{2}+C^{\prime}$ have the same length $l\left(W_{1}\right)=l\left(W_{2}\right) \leq 2 n-2$ and different signs. As the proof of Subcase 1.1, we can construct an even walk $W$ with length $l(W) \leq 2 n-2-l$. So there exists a pair of SSSD walks from $x$ to $y$ of length $2 n-2$. It remains to consider the following subcase.

Subcase 2.2. $C$ and $C^{\prime}$ have at least one common vertex.

Let $G_{3}=C \cup C^{\prime}$. Let $x$ and $y$ be two arbitrary (not necessarily distinct) vertices in $S(A)$. Suppose that $P_{1}$ is the shortest path from $x$ to $G_{3}$ and intersects $G_{3}$ at vertex $x^{\prime}$ and $P_{2}$ is the shortest path from $y$ to $G_{3}$ and intersects $G_{3}$ at vertex $y^{\prime}$. Denote $C \cap C^{\prime}$ by $R$. Assume $R$ has $m$ vertices, where $1 \leq m \leq \min \left(l_{1}, l_{2}\right)$, then

$$
0 \leq l\left(P_{1}\right), \quad l\left(P_{2}\right) \leq n-l_{1}-l_{2}+m .
$$

If $m>1$, then $R$ is a path with vertex set $V(R)=\left\{v_{1}, v_{2}, \ldots, v_{m}\right\}$ and edge set $E(R)=$ $\left\{\left(v_{1}, v_{2}\right),\left(v_{2}, v_{3}\right), \ldots,\left(v_{m-1}, v_{m}\right)\right\}$. If $m=l^{\prime}$, since there exists no negative even cycles in $S(A)$, then $C$ and $C^{\prime}$ have the same sign, a contradiction. Therefore, $1 \leq m<l^{\prime}$.

Subcase 2.2.1. $x^{\prime} \in C \backslash R$ and $y^{\prime} \in C \backslash R$. See Figure 3(a).

Without loss of generality, we assume that $l\left(Q_{C \backslash R}\left(x^{\prime} \rightarrow v_{1}\right)\right) \leq l\left(Q_{C \backslash R}\left(y^{\prime} \rightarrow v_{1}\right)\right)$. Let $w=l\left(P_{1}\right)+l\left(Q_{C \backslash R}\left(x^{\prime} \rightarrow v_{1}\right)\right)+l\left(Q_{R}\left(v_{1} \rightarrow v_{m}\right)\right)+l\left(Q_{C \backslash R}\left(v_{m} \rightarrow y^{\prime}\right)\right)+l\left(P_{2}\right)$. If $w$ is odd, set

$$
W^{\prime}=P_{1}+Q_{C \backslash R}\left(x^{\prime} \longrightarrow v_{1}\right)+Q_{R}\left(v_{1} \longrightarrow v_{m}\right)+Q_{C \backslash R}\left(v_{m} \longrightarrow y^{\prime}\right)+P_{2}
$$

Otherwise, set

$$
W^{\prime \prime}=P_{1}+Q_{C \backslash R}\left(x^{\prime} \longrightarrow v_{1}\right)+Q_{C^{\prime} \backslash R}\left(v_{1} \longrightarrow v_{m}\right)+Q_{C \backslash R}\left(v_{m} \longrightarrow y^{\prime}\right)+P_{2} .
$$


Let

$$
W_{1}=\left\{\begin{array}{ll}
W^{\prime}+C, & w \text { is odd }, \\
W^{\prime \prime}+C, & \text { otherwise }
\end{array} \quad W_{2}= \begin{cases}W^{\prime}+C^{\prime}+\frac{l-l^{\prime}}{2} C_{2}, & w \text { is odd } \\
W^{\prime \prime}+C^{\prime}+\frac{l-l^{\prime}}{2} C_{2}, & \text { otherwise }\end{cases}\right.
$$

where $C_{2}$ is a positive 2-cycle that contains $x$. Since $C^{\prime}$ is odd, $l\left(Q_{R}\left(v_{1} \rightarrow v_{m}\right)\right)$ and $l\left(Q_{C^{\prime} \backslash R}\left(v_{1} \rightarrow v_{m}\right)\right)$ have different parity. Therefore, both $l\left(W_{1}\right)$ and $l\left(W_{2}\right)$ are even. Then, if $w$ is odd,

$$
l\left(W_{1}\right)=l\left(W_{2}\right) \leq 2\left(n-l-l^{\prime}+m\right)+l+l=2 n-2 l^{\prime}+2 m .
$$

Otherwise,

$$
l\left(W_{1}\right)=l\left(W_{2}\right) \leq 2\left(n-l-l^{\prime}+m\right)+(l-m+1)+\left(l^{\prime}-m+1\right)+l=2 n-l^{\prime}+2 .
$$

Since both $l\left(W_{1}\right)$ and $l\left(W_{2}\right)$ are even, it follows that $l\left(W_{1}\right)=l\left(W_{2}\right) \leq 2 n-2$. We see that the pair $W_{1}, W_{2}$ is a pair of SSSD walks with even length. Therefore, there exists a pair of SSSD walks from $x$ to $y$ with length $2 n-2$.

Subcase 2.2.2. $x^{\prime} \in C \backslash R$ and $y^{\prime} \in R$. See Figure 3(b).

Let $w=l\left(P_{1}\right)+l\left(Q_{C}\left(x^{\prime} \rightarrow y^{\prime}\right)\right)+l\left(P_{2}\right)$. If $w$ is odd, set

$$
W^{\prime}=P_{1}+Q_{C}\left(x^{\prime} \longrightarrow y^{\prime}\right)+P_{2} .
$$

Otherwise, set

$$
W^{\prime \prime}=P_{1}+C \backslash Q_{C}\left(x^{\prime} \longrightarrow y^{\prime}\right)+P_{2}
$$

Let

$$
W_{1}=\left\{\begin{array}{ll}
W^{\prime}+C, & w \text { is odd }, \\
W^{\prime \prime}+C, & \text { otherwise }
\end{array} \quad W_{2}= \begin{cases}W^{\prime}+C^{\prime}+\frac{l-l^{\prime}}{2} C_{2}, & w \text { is odd }, \\
W^{\prime \prime}+C^{\prime}+\frac{l-l^{\prime}}{2} C_{2}, & \text { otherwise }\end{cases}\right.
$$

where $C_{2}$ is a positive 2-cycle that contains $x$. Since $C$ is odd, $C \backslash Q_{C}\left(x^{\prime} \rightarrow y^{\prime}\right)$ and $Q_{C}\left(x^{\prime} \rightarrow\right.$ $\left.y^{\prime}\right)$ have different parity, Therefore, both $l\left(W_{1}\right)$ and $l\left(W_{2}\right)$ are even. Then, if $w$ is odd,

$$
l\left(W_{1}\right)=l\left(W_{2}\right) \leq 2\left(n-l-l^{\prime}+m\right)+\frac{l-1}{2}+l=2 n-2 l^{\prime}+2 m-\frac{l+1}{2} .
$$

Otherwise,

$$
l\left(W_{1}\right)=l\left(W_{2}\right) \leq 2\left(n-l-l^{\prime}+m\right)+(l-1)+l=2 n-2 l^{\prime}+2 m-1 .
$$


Since both $l\left(W_{1}\right)$ and $l\left(W_{2}\right)$ are even, it follows that $l\left(W_{1}\right)=l\left(W_{2}\right) \leq 2 n-2$. We see that the pair $W_{1}, W_{2}$ is a pair of SSSD walks with even length. Therefore, there exists a pair of SSSD walks from $x$ to $y$ with length $2 n-2$.

Subcase 2.2.3. $x^{\prime} \in C \backslash R$ and $y^{\prime} \in C^{\prime} \backslash R$. See Figure 3(c).

Without loss of generality, we assume that $l\left(Q_{C \backslash R}\left(x^{\prime} \rightarrow v_{1}\right)\right) \leq l\left(Q_{C \backslash R}\left(x^{\prime} \rightarrow v_{m}\right)\right)$. Let $w=l\left(P_{1}\right)+l\left(Q_{C \backslash R}\left(x^{\prime} \rightarrow v_{1}\right)\right)+l\left(Q_{C^{\prime} \backslash R}\left(v_{1} \rightarrow y^{\prime}\right)\right)+l\left(P_{2}\right)$. If $w$ is odd, set

$$
W^{\prime}=P_{1}+Q_{C \backslash R}\left(x^{\prime} \longrightarrow v_{1}\right)+Q_{C^{\prime} \backslash R}\left(v_{1} \longrightarrow y^{\prime}\right)+P_{2}
$$

Otherwise, set

$$
W^{\prime \prime}=P_{1}+Q_{C \backslash R}\left(x^{\prime} \longrightarrow v_{1}\right)+Q_{R}\left(v_{1} \longrightarrow v_{m}\right)+Q_{C^{\prime} \backslash R}\left(v_{m} \longrightarrow y^{\prime}\right)+P_{2}
$$

Let

$$
W_{1}=\left\{\begin{array}{ll}
W^{\prime}+C, & w \text { is odd }, \\
W^{\prime \prime}+C, & \text { otherwise, }
\end{array} \quad W_{2}= \begin{cases}W^{\prime}+C^{\prime}+\frac{l-l^{\prime}}{2} C_{2}, & w \text { is odd } \\
W^{\prime \prime}+C^{\prime}+\frac{l-l^{\prime}}{2} C_{2}, & \text { otherwise }\end{cases}\right.
$$

where $C_{2}$ is a positive 2-cycle that contains $x$. Since $C^{\prime}$ is odd, $l\left(Q_{C^{\prime} \backslash R}\left(v_{1} \rightarrow y^{\prime}\right)\right)$ and $l\left(Q_{R}\left(v_{1} \rightarrow v_{m}\right)\right)+l\left(Q_{C^{\prime} \backslash R}\left(v_{m} \rightarrow y^{\prime}\right)\right)$ have different parity. Therefore, both $l\left(W_{1}\right)$ and $l\left(W_{2}\right)$ are even. Noting that $W_{3}=P_{1}+Q_{C \backslash R}\left(x^{\prime} \rightarrow v_{1}\right)+Q_{C^{\prime} \backslash R}\left(v_{1} \rightarrow y^{\prime}\right)$ and $W_{4}=P_{1}+Q_{C \backslash R}\left(x^{\prime} \rightarrow\right.$ $\left.v_{1}\right)+Q_{R}\left(v_{1} \rightarrow v_{m}\right)+Q_{C^{\prime} \backslash R}\left(v_{m} \rightarrow y^{\prime}\right)$ are paths of $S(A)$, then we have $l\left(W_{3}\right), l\left(W_{4}\right) \leq n-1$. Therefore,

$$
l\left(W_{1}\right)=l\left(W_{2}\right) \leq(n-1)+\left(n-l-l^{\prime}+m\right)+l=2 n-l^{\prime}+m-1 .
$$

Since both $l\left(W_{1}\right)$ and $l\left(W_{2}\right)$ are even, it follows that $l\left(W_{1}\right)=l\left(W_{2}\right) \leq 2 n-2$. We see that the pair $W_{1}, W_{2}$ is a pair of SSSD walks with even length. Therefore, there exists a pair of SSSD walks from $x$ to $y$ with length $2 n-2$.

Subcase 2.2.4. $x^{\prime} \in R$ and $y^{\prime} \in R$. See Figure 3(d).

The proof for this subcase is similar to that of Subcase 2.2.2, so we omit it.

Subcase 2.2.5. $x^{\prime} \in R$ and $y^{\prime} \in C^{\prime} \backslash R$. See Figure 3(e).

The proof for this subcase is similar to that of Subcase 2.2.2, so we omit it.

Subcase 2.2.6. $x^{\prime} \in C^{\prime} \backslash R$ and $y^{\prime} \in C^{\prime} \backslash R$. See Figure 3(f).

The proof for this subcase is similar to that of Subcase 2.2.1, so we omit it.

From all the above subcases, there exists a pair of SSSD walks from $x$ to $y$ with length $2 n-2$. Therefore, we get $l(A) \leq 2 n-2$ by Lemma 2.6 .

Lemma 3.2. Let $A$ be an $n \times n$ primitive nonpowerful ZS sign pattern matrix with zero diagonal. If there exist a negative 2-cycle and a positive 2-cycle in $S(A)$, then $l(A) \leq 2 n-2$. 


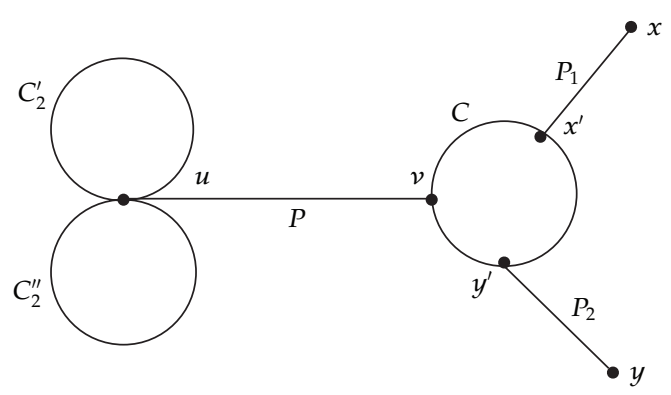

Figure 5: Illustration for Case 3 of Lemma 3.2.

Proof. Since $A$ is primitive, it follows from Lemma 2.4 that $S(A)$ is strongly connected and there is an odd cycle $C$ in $S(A)$ with length $l(C)=l$. Since $A$ has zero diagonal, there are no loops in $S(A)$ and so $l \geq 3$. Without loss of generality, we assume that $C$ is an odd cycle with the least length in $S(A)$. Since $A$ is $\mathrm{ZS}$ and $S(A)$ contain a positive 2-cycle and a negative 2cycle, there exists a positive 2-cycle $C_{2}^{\prime}$ and a negative 2-cycle $C_{2}^{\prime \prime}$ such that $V\left(C_{2}^{\prime}\right) \cap V\left(C_{2}^{\prime \prime}\right) \neq \emptyset$. Let $u \in V\left(C_{2}^{\prime}\right) \cap V\left(C_{2}^{\prime \prime}\right)$.

Let $P$ be the shortest path from $u$ to $C$. Let $x$ and $y$ be two arbitrary (not necessarily distinct) vertices in $S(A)$. Suppose there are $k$ vertices on $P$ and $P$ intersects $C$ at $v$, where $k \geq 1$. Suppose $P_{1}$ is the shortest path from $x$ to $P \cup C$ and $P_{1}$ intersects $P \cup C$ at $x^{\prime}$ and $P_{2}$ is the shortest path from $y$ to $P \cup C$ and $P_{2}$ intersects $P \cup C$ at $y^{\prime}$ where $0 \leq l\left(P_{1}\right), l\left(P_{2}\right) \leq n-l-k+1$. We consider the following three cases.

Case 1. $x^{\prime} \in P$ and $y^{\prime} \in P$.

By the Subcase 2.1 of Lemma 4.2 in [3], there exists a pair of SSSD walks from $x$ to $y$ of length $2 n-2$.

Case 2. Only one of $x^{\prime}$ and $y^{\prime}$ belongs to $P$.

By the Subcase 2.2 of Lemma 4.2 in [3], there exists a pair of SSSD walks from $x$ to $y$ of length $2 n-2$.

It remains to consider the following case.

Case 3. $x^{\prime} \notin P$ and $y^{\prime} \notin P$. See Figure 5 .

Subcase 3.1. $x^{\prime}=y^{\prime}$.

Let $w=l\left(P_{1}\right)+l\left(Q_{C}\left(x^{\prime} \rightarrow v\right)\right)+2 l(P)+l\left(Q_{C}\left(v \rightarrow y^{\prime}\right)\right)+l\left(P_{2}\right)$. If $w$ is even, set

$$
W^{\prime}=P_{1}+Q_{C}\left(x^{\prime} \longrightarrow v\right)+2 P+Q_{C}\left(v \longrightarrow y^{\prime}\right)+P_{2} .
$$

Otherwise, set

$$
W^{\prime \prime}=P_{1}+Q_{C}\left(x^{\prime} \longrightarrow v\right)+2 P+C \backslash Q_{C}\left(v \longrightarrow y^{\prime}\right)+P_{2}
$$


Let

$$
W_{1}=\left\{\begin{array}{ll}
W^{\prime}+C_{2}^{\prime}, & w \text { is even, } \\
W^{\prime \prime}+C_{2}^{\prime}, & \text { otherwise },
\end{array} \quad W_{2}= \begin{cases}W^{\prime}+C_{2}^{\prime \prime} & w \text { is even } \\
W^{\prime \prime}+C_{2}^{\prime \prime}, & \text { otherwise }\end{cases}\right.
$$

Since $C$ is odd, $l\left(Q_{C}\left(v \rightarrow x^{\prime}\right)\right)$ and $l\left(C \backslash Q_{C}\left(v \rightarrow x^{\prime}\right)\right)$ have different parity. Therefore, both $l\left(W_{1}\right)$ and $l\left(W_{2}\right)$ are even. Then, if $w$ is odd,

$$
l\left(W_{1}\right)=l\left(W_{2}\right) \leq 2(n-l-k+1)+2(k-1)+(l-1)+2=2 n-l+1 .
$$

Otherwise,

$$
l\left(W_{1}\right)=l\left(W_{2}\right) \leq 2(n-l-k+1)+2(k-1)+l+2=2 n-l+2 .
$$

Since both $l\left(W_{1}\right)$ and $l\left(W_{2}\right)$ are even, it follows that $l\left(W_{1}\right)=l\left(W_{2}\right) \leq 2 n-2$. We see that the pair $W_{1}, W_{2}$ is a pair of SSSD walks with even length. Therefore, there exists a pair of SSSD walks from $x$ to $y$ with length $2 n-2$.

Subcase 3.2. $x^{\prime} \neq y^{\prime}$.

Without loss of generality, we assume that $l\left(Q_{C}\left(x^{\prime} \rightarrow v\right)\right) \leq l\left(Q_{C}\left(y^{\prime} \rightarrow v\right)\right)$. Let $P_{3}=Q_{C}\left(x^{\prime} \rightarrow v\right)$ and $P_{4}=Q_{C \backslash P_{3}}\left(v \rightarrow y^{\prime}\right)$. Let $w=l\left(P_{1}\right)+l\left(P_{3}\right)+l\left(P_{4}\right)+2 l(P)+l\left(P_{2}\right)$, If $w$ is odd, set

$$
W^{\prime}=P_{1}+Q_{C}\left(x^{\prime} \longrightarrow v\right)+2 P+P_{4}+P_{2} .
$$

Otherwise, set

$$
W^{\prime \prime}=P_{1}+Q_{C}\left(x^{\prime} \longrightarrow v\right)+2 P+Q_{C}\left(v \longrightarrow x^{\prime}\right)+Q_{C \backslash\left\{P_{3}+P_{4}\right\}}\left(x^{\prime} \longrightarrow y^{\prime}\right)+P_{2}
$$

Let

$$
W_{1}=\left\{\begin{array}{ll}
W^{\prime}+C_{2}^{\prime}, & w \text { is even, } \\
W^{\prime \prime}+C_{2}^{\prime}, & \text { otherwise, }
\end{array} \quad W_{2}= \begin{cases}W^{\prime}+C_{2}^{\prime \prime}, & w \text { is even } \\
W^{\prime \prime}+C_{2}^{\prime \prime}, & \text { otherwise }\end{cases}\right.
$$

Since $C$ is odd, $l\left(P_{3}\right)+l\left(P_{4}\right)$ and $l\left(Q_{C \backslash\left\{P_{3}+P_{4}\right\}}\left(x^{\prime} \rightarrow y^{\prime}\right)\right)$ have different parity. Therefore, both $l\left(W_{1}\right)$ and $l\left(W_{2}\right)$ are even. Then, if $w$ is odd,

$$
l\left(W_{1}\right)=l\left(W_{2}\right) \leq 2(n-l-k+1)+2(k-1)+(l-1)+2=2 n-l+1 .
$$

Otherwise,

$$
l\left(W_{1}\right)=l\left(W_{2}\right) \leq 2(n-l-k+1)+2(k-1)+(l-1)+(l-2)+2=2 n-1 .
$$


Since both $l\left(W_{1}\right)$ and $l\left(W_{2}\right)$ are even, it follows that $l\left(W_{1}\right)=l\left(W_{2}\right) \leq 2 n-2$. We see that the pair $W_{1}, W_{2}$ is a pair of SSSD walks with even length. Therefore, there exists a pair of SSSD walks from $x$ to $y$ with length $2 n-2$.

Therefore, $l(A) \leq 2 n-2$ by Lemma 2.6 .

Lemma 3.3. Let $A$ be an $n \times n$ primitive nonpowerful ZS sign pattern matrix with zero diagonal. If $l(A)=2 n-1$, then all 2 -cycles in $S(A)$ are negative.

Proof. Assume that $l(A)=2 n-1$. By Lemma 3.1, $S(A)$ has at least one negative 2-cycle. It then follows from Lemma 3.2 that all 2-cycles in $S(A)$ are negative.

Lemma 3.4. Let $A$ be an $n \times n$ primitive nonpowerful ZS sign pattern matrix with zero diagonal. Suppose that $l(A)=2 n-1$. Then there exists an odd cycle $C$ in $S(A)$ and $C$ is the only cycle of length at least 3 in $S(A)$.

Proof. Since $A$ is primitive, it follows from Lemma 2.4 that $S(A)$ is strongly connected and there is an odd cycle $C=\left(v_{1}, v_{2}, \ldots, v_{l}\right)$ in $S(A)$ with length $l(C)=l$. Since $A$ has zero diagonal, there is no loop in $S(A)$ and so $l \geq 3$. Consider the following two directed cycles:

$$
C^{1}=\left(v_{1}, v_{2}, \ldots, v_{l}, v_{1}\right), \quad C^{2}=\left(v_{1}, v_{l}, \ldots, v_{2}, v_{1}\right)
$$

Since there exists no positive 2-cycle in $S(A)$ by Lemma 3.3, the $\operatorname{arcs}\left(v_{i}, v_{i+1}\right)$ and $\left(v_{i+1}, v_{i}\right)$ have different signs. Thus $\operatorname{sgn}\left(C^{1}\right)=-\operatorname{sgn}\left(C^{2}\right)$ by the fact that $l$ is odd. Without loss of generality, we assume that $C$ is an odd cycle with the least length in $S(A)$.

If $l(A)=2 n-1$, suppose there exists another cycle $C^{\prime}$ with length $l\left(C^{\prime}\right)=l^{\prime} \geq 3$, we consider the following two cases.

Case 1. $C$ and $C^{\prime}$ have no common vertices.

Let $P$ be the shortest path from $C$ to $C^{\prime}$. Suppose $P$ intersects $C$ at vertex $u$ and intersects $C^{\prime}$ at vertex $v$ and there are $k$ vertices on $P$ where $k \geq 2$. Let $G_{4}=C \cup P \cup C^{\prime}$. Let $x$ and $y$ be two arbitrary (not necessarily distinct) vertices in $S(A)$. Suppose that $P_{1}$ is the shortest path from $x$ to $G_{4}$ and intersects $G_{4}$ at vertex $x^{\prime}$ and $P_{2}$ is the shortest path from $y$ to $G_{4}$ and intersects $G_{4}$ at vertex $y^{\prime}$. Then

$$
0 \leq l\left(P_{1}\right), \quad l\left(P_{2}\right) \leq n-l-l^{\prime}-k+2 .
$$

We consider the following six subcases.

Subcase 1.1. $x^{\prime} \in C$ and $y^{\prime} \in C$. See Figure 6(a).

$$
\begin{aligned}
& \text { Let } w=l\left(P_{1}\right)+l\left(Q_{C}\left(x^{\prime} \rightarrow y^{\prime}\right)\right)+l\left(P_{2}\right) \text {. If } w \text { is odd, set } \\
& \qquad W^{\prime}=P_{1}+Q_{C}\left(x^{\prime} \longrightarrow y^{\prime}\right)+P_{2} .
\end{aligned}
$$

Otherwise, set

$$
W^{\prime \prime}=P_{1}+C \backslash Q_{C}\left(x^{\prime} \longrightarrow y^{\prime}\right)+P_{2} .
$$




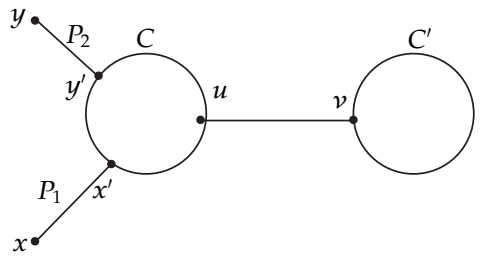

(a)

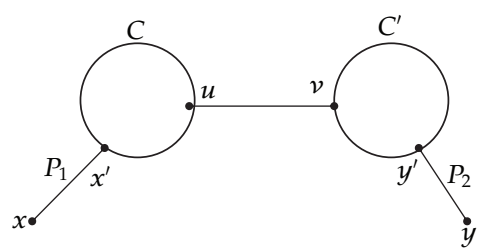

(c)

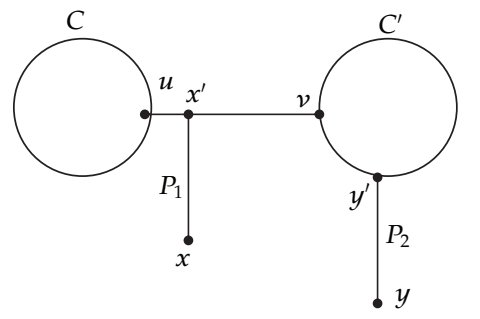

(e)

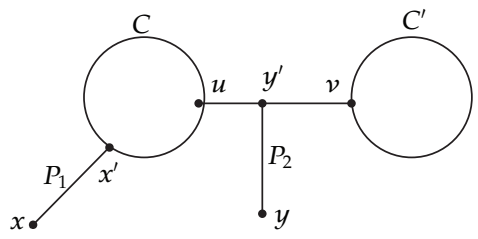

(b)

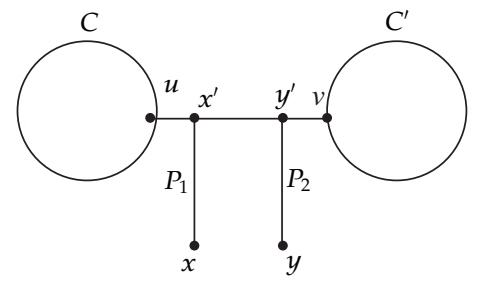

(d)

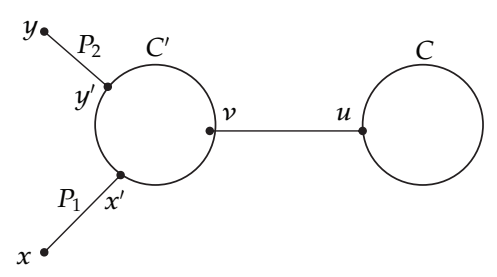

(f)

Figure 6: Illustration for Lemma 3.4.

Let

$$
W_{1}=\left\{\begin{array}{ll}
W^{\prime}+C^{1}, & w \text { is odd }, \\
W^{\prime \prime}+C^{1}, & \text { otherwise },
\end{array} \quad W_{2}= \begin{cases}W^{\prime}+C^{2}, & w \text { is odd } \\
W^{\prime \prime}+C^{2}, & \text { otherwise }\end{cases}\right.
$$

Since $C$ is odd, $l\left(Q_{C}\left(x^{\prime} \rightarrow y^{\prime}\right)\right)$ and $l\left(C \backslash Q_{C}\left(x^{\prime} \rightarrow y^{\prime}\right)\right)$ have different parity. Therefore, both $l\left(W_{1}\right)$ and $l\left(W_{2}\right)$ are even. Then, if $w$ is odd,

$$
l\left(W_{1}\right)=l\left(W_{2}\right) \leq 2\left(n-l-l^{\prime}-k+2\right)+\frac{l-1}{2}+l=2 n-2 l^{\prime}-2 k-\frac{l}{2}+\frac{7}{2} .
$$

Otherwise,

$$
l\left(W_{1}\right)=l\left(W_{2}\right) \leq 2\left(n-l-l^{\prime}-k+2\right)+l+l=2 n-2 l^{\prime}-2 k+4 .
$$

Since both $l\left(W_{1}\right)$ and $l\left(W_{2}\right)$ are even, it follows that $l\left(W_{1}\right)=l\left(W_{2}\right) \leq 2 n-2$. We see that the pair $W_{1}, W_{2}$ is a pair of SSSD walks with even length. Therefore, there exists a pair of SSSD walks from $x$ to $y$ with length $2 n-2$.

Subcase 1.2. $x^{\prime} \in C$ and $y^{\prime} \in P$. See Figure 6(b). 
Journal of Applied Mathematics

$$
\begin{gathered}
\text { Let } W=l\left(P_{1}\right)+l\left(Q_{C}\left(x^{\prime} \rightarrow u\right)\right)+l\left(Q_{P}\left(u \rightarrow y^{\prime}\right)\right)+l\left(P_{2}\right) \text {. If } w \text { is odd, set } \\
\qquad W^{\prime}=P_{1}+Q_{C}\left(x^{\prime} \longrightarrow u\right)+Q_{P}\left(u \longrightarrow y^{\prime}\right)+P_{2} .
\end{gathered}
$$

Otherwise, set

$$
W^{\prime}=P_{1}+C \backslash Q_{C}\left(x^{\prime} \longrightarrow u\right)+Q_{P}\left(u \longrightarrow y^{\prime}\right)+P_{2}
$$

Let

$$
W_{1}=\left\{\begin{array}{ll}
W^{\prime}+C^{1}, & w \text { is odd }, \\
W^{\prime \prime}+C^{1}, & \text { otherwise },
\end{array} \quad W_{2}= \begin{cases}W^{\prime}+C^{2}, & w \text { is odd } \\
W^{\prime \prime}+C^{2}, & \text { otherwise }\end{cases}\right.
$$

Since $C$ is odd, $l\left(Q_{C}\left(x^{\prime} \rightarrow u\right)\right)$ and $l\left(C \backslash Q_{C}\left(x^{\prime} \rightarrow u\right)\right)$ have different parity. Therefore, both $l\left(W_{1}\right)$ and $l\left(W_{2}\right)$ are even. Then, if $w$ is odd,

$$
l\left(W_{1}\right)=l\left(W_{2}\right) \leq 2\left(n-l-l^{\prime}-k+2\right)+\frac{l-1}{2}+(k-1)+l=2 n-2 l^{\prime}-k-\frac{l}{2}+\frac{5}{2} .
$$

Otherwise,

$$
l\left(W_{1}\right)=l\left(W_{2}\right) \leq 2\left(n-l-l^{\prime}-k+2\right)+(k-1)+l+l=2 n-2 l^{\prime}-k+3 .
$$

Since both $l\left(W_{1}\right)$ and $l\left(W_{2}\right)$ are even, it follows that $l\left(W_{1}\right)=l\left(W_{2}\right) \leq 2 n-2$. We see that the pair $W_{1}, W_{2}$ is a pair of SSSD walks with even length. Therefore, there exists a pair of SSSD walks from $x$ to $y$ with length $2 n-2$.

Subcase 1.3. $x^{\prime} \in C$ and $y^{\prime} \in C^{\prime}$. See Figure 6(c).

Let $w=l\left(P_{1}\right)+l\left(Q_{C}\left(x^{\prime} \rightarrow u\right)\right)+l(P)+l\left(Q_{C^{\prime}}\left(v \rightarrow y^{\prime}\right)\right)+l\left(P_{2}\right)$. If $w$ is odd, set

$$
W^{\prime}=P_{1}+Q_{C}\left(x^{\prime} \longrightarrow u\right)+P+Q_{C^{\prime}}\left(v \longrightarrow y^{\prime}\right)+P_{2} .
$$

Otherwise, set

$$
W^{\prime \prime}=P_{1}+C \backslash Q_{C}\left(x^{\prime} \longrightarrow u\right)+P+Q_{C^{\prime}}\left(v \longrightarrow y^{\prime}\right)+P_{2}
$$

Let

$$
W_{1}=\left\{\begin{array}{ll}
W^{\prime}+C^{1}, & w \text { is odd }, \\
W^{\prime \prime}+C^{1}, & \text { otherwise },
\end{array} \quad W_{2}= \begin{cases}W^{\prime}+C^{2}, & w \text { is odd } \\
W^{\prime \prime}+C^{2}, & \text { otherwise }\end{cases}\right.
$$


Since $C$ is odd, $l\left(Q_{C}\left(x^{\prime} \rightarrow u\right)\right)$ and $l\left(C \backslash Q_{C}\left(x^{\prime} \rightarrow u\right)\right)$ have different parity. Therefore, both $l\left(W_{1}\right)$ and $l\left(W_{2}\right)$ are even. Then, if $w$ is odd,

$$
l\left(W_{1}\right)=l\left(W_{2}\right) \leq 2\left(n-l-l^{\prime}-k+2\right)+\frac{l-1}{2}+(k-1)+\frac{l^{\prime}}{2}+l=2 n-k-\frac{l}{2}-\frac{3 l^{\prime}}{2}+\frac{5}{2} .
$$

Otherwise,

$$
l\left(W_{1}\right)=l\left(W_{2}\right) \leq 2\left(n-l-l^{\prime}-k+2\right)+l+(k-1)+\frac{l^{\prime}}{2}+l=2 n-k-\frac{3 l^{\prime}}{2}+3
$$

Since both $l\left(W_{1}\right)$ and $l\left(W_{2}\right)$ are even, it follows that $l\left(W_{1}\right)=l\left(W_{2}\right) \leq 2 n-2$. We see that the pair $W_{1}, W_{2}$ is a pair of SSSD walks with even length. Therefore, there exists a pair of SSSD walks from $x$ to $y$ with length $2 n-2$.

Subcase 1.4. $x^{\prime} \in P$ and $y^{\prime} \in P$. See Figure 6(d).

Without loss of generality, we assume that $l\left(Q_{P}\left(x^{\prime} \rightarrow u\right)\right) \leq l\left(Q_{P}\left(y^{\prime} \rightarrow u\right)\right)$. Let $w=l\left(P_{1}\right)+l\left(Q_{P}\left(x^{\prime} \rightarrow u\right)\right)+l(P)+l\left(Q_{P}\left(v \rightarrow y^{\prime}\right)\right)+l\left(P_{2}\right)$. If $w$ is odd, set

$$
W^{\prime}=P_{1}+Q_{P}\left(x^{\prime} \longrightarrow u\right)+P+Q_{P}\left(v \longrightarrow y^{\prime}\right)+P_{2}
$$

Otherwise, set

$$
W^{\prime \prime}=P_{1}+Q_{P}\left(x^{\prime} \longrightarrow u\right)+P+Q_{P}\left(v \longrightarrow y^{\prime}\right)+P_{2}+C .
$$

Let

$$
W_{1}=\left\{\begin{array}{ll}
W^{\prime}+C^{1}, & w \text { is odd }, \\
W^{\prime \prime}+C^{1}, & \text { otherwise },
\end{array} \quad W_{2}= \begin{cases}W^{\prime}+C^{2}, & w \text { is odd } \\
W^{\prime \prime}+C^{2}, & \text { otherwise }\end{cases}\right.
$$

Therefore, both $l\left(W_{1}\right)$ and $l\left(W_{2}\right)$ are even. Then, if $w$ is odd,

$$
l\left(W_{1}\right)=l\left(W_{2}\right) \leq 2\left(n-l-l^{\prime}-k+2\right)+2(k-1)+l=2 n-l-2 l^{\prime}+2 .
$$

Otherwise,

$$
l\left(W_{1}\right)=l\left(W_{2}\right) \leq 2\left(n-l-l^{\prime}-k+2\right)+2(k-1)+l+l=2 n-2 l^{\prime}+2 .
$$

Since both $l\left(W_{1}\right)$ and $l\left(W_{2}\right)$ are even, it follows that $l\left(W_{1}\right)=l\left(W_{2}\right) \leq 2 n-2$. We see that the pair $W_{1}, W_{2}$ is a pair of SSSD walks with even length. Therefore, there exists a pair of SSSD walks from $x$ to $y$ with length $2 n-2$.

Subcase 1.5. $x^{\prime} \in P$ and $y^{\prime} \in C^{\prime}$. See Figure 6(e).

Let $w=l\left(P_{1}\right)+l\left(Q_{P}\left(x^{\prime} \rightarrow u\right)\right)+l(P)+l\left(Q_{C^{\prime}}\left(v \rightarrow y^{\prime}\right)\right)+l\left(P_{2}\right)$, if $w$ is odd, set

$$
W^{\prime}=P_{1}+Q_{P}\left(x^{\prime} \longrightarrow u\right)+P+Q_{C^{\prime}}\left(v \longrightarrow y^{\prime}\right)+P_{2}
$$


Otherwise, set

$$
W^{\prime \prime}=P_{1}+Q_{P}\left(x^{\prime} \longrightarrow u\right)+P+Q_{C^{\prime}}\left(v \longrightarrow y^{\prime}\right)+P_{2}+C .
$$

Let

$$
W_{1}=\left\{\begin{array}{ll}
W^{\prime}+C^{1}, & w \text { is odd }, \\
W^{\prime \prime}+C^{1}, & \text { otherwise }
\end{array} \quad W_{2}= \begin{cases}W^{\prime}+C^{2}, & w \text { is odd } \\
W^{\prime \prime}+C^{2}, & \text { otherwise }\end{cases}\right.
$$

Since $C$ is odd, both $l\left(W_{1}\right)$ and $l\left(W_{2}\right)$ are even. Then, if $w$ is odd,

$$
l\left(W_{1}\right)=l\left(W_{2}\right) \leq 2\left(n-l-l^{\prime}-k+2\right)+2(k-1)+\frac{l^{\prime}}{2}+l=2 n-l-\frac{3 l^{\prime}}{2}+2 .
$$

Otherwise,

$$
l\left(W_{1}\right)=l\left(W_{2}\right) \leq 2\left(n-l-l^{\prime}-k+2\right)+2(k-1)+\frac{l^{\prime}}{2}+l+l=2 n-\frac{3 l^{\prime}}{2}+2 .
$$

Since both $l\left(W_{1}\right)$ and $l\left(W_{2}\right)$ are even, it follows that $l\left(W_{1}\right)=l\left(W_{2}\right) \leq 2 n-2$. We see that the pair $W_{1}, W_{2}$ is a pair of SSSD walks with even length. Therefore, there exists a pair of SSSD walks from $x$ to $y$ with length $2 n-2$.

Subcase 1.6. $x^{\prime} \in C^{\prime}$ and $y^{\prime} \in C^{\prime}$. See Figure 6(f).

Let $w=l\left(P_{1}\right)+l\left(Q_{C^{\prime}}\left(x^{\prime} \rightarrow v\right)\right)+l\left(Q_{C^{\prime}}\left(v \rightarrow y^{\prime}\right)\right)+2 l(P)+l\left(P_{2}\right)$. If $w$ is odd, set

$$
W^{\prime}=P_{1}+Q_{C^{\prime}}\left(x^{\prime} \longrightarrow v\right)+2 P+Q_{C^{\prime}}\left(v \longrightarrow y^{\prime}\right)+P_{2} .
$$

Otherwise, set

$$
W^{\prime \prime}=P_{1}+Q_{C^{\prime}}\left(x^{\prime} \longrightarrow v\right)+2 P+Q_{C^{\prime}}\left(v \longrightarrow y^{\prime}\right)+P_{2}+C .
$$

Let

$$
W_{1}=\left\{\begin{array}{ll}
W^{\prime}+C^{1}, & w \text { is odd }, \\
W^{\prime \prime}+C^{1}, & \text { otherwise },
\end{array} \quad W_{2}= \begin{cases}W^{\prime}+C^{2}, & w \text { is odd } \\
W^{\prime \prime}+C^{2}, & \text { otherwise }\end{cases}\right.
$$

Since $C$ is odd, both $l\left(W_{1}\right)$ and $l\left(W_{2}\right)$ are even. Then if $w$ is odd,

$$
l\left(W_{1}\right)=l\left(W_{2}\right) \leq 2\left(n-l-l^{\prime}-k+2\right)+2(k-1)+l^{\prime}+l=2 n-l-l^{\prime}+2 .
$$

Otherwise,

$$
l\left(W_{1}\right)=l\left(W_{2}\right) \leq 2\left(n-l-l^{\prime}-k+2\right)+2(k-1)+l^{\prime}+l+l=2 n-l^{\prime}+2 .
$$


Since both $l\left(W_{1}\right)$ and $l\left(W_{2}\right)$ are even, it follows that $l\left(W_{1}\right)=l\left(W_{2}\right) \leq 2 n-2$. We see that the pair $W_{1}, W_{2}$ is a pair of SSSD walks with even length. Therefore, there exists a pair of SSSD walks from $x$ to $y$ with length $2 n-2$.

Therefore, $l(A) \leq 2 n-2$ by Lemma 2.6 , a contradiction.

Case 2. $C$ and $C^{\prime}$ have at least one common vertex.

Let $G_{5}=C \cup C^{\prime}$. Let $x$ and $y$ be two arbitrary (not necessarily distinct) vertices in $S(A)$. Suppose that $P_{1}$ is the shortest path from $x$ to $G_{5}$ and intersects $G_{5}$ at vertex $x^{\prime}$ and $P_{2}$ is the shortest path from $y$ to $G_{5}$ and intersects $G_{5}$ at vertex $y^{\prime}$. Denote $C \cap C^{\prime}$ by $R$. Assume $R$ has $m$ vertices, where $1 \leq m \leq \min \left(l_{1}, l_{2}\right)$, then

$$
0 \leq l\left(P_{1}\right), \quad l\left(P_{2}\right) \leq n-l_{1}-l_{2}+m .
$$

If $m>1$, then $R$ is a path with vertex set $V(R)=\left\{v_{1}, v_{2}, \ldots, v_{m}\right\}$ and edge set $E(R)=$ $\left\{\left(v_{1}, v_{2}\right),\left(v_{2}, v_{3}\right), \ldots,\left(v_{m-1}, v_{m}\right)\right\}$. If $l^{\prime}$ is odd, $m=\min \left(l, l^{\prime}\right)=l$ by the minimality of $C$. If $l^{\prime}$ is even, suppose $\min \left(l, l^{\prime}\right)=l^{\prime}$, then there exists an odd cycle $C^{\prime}$ with $l\left(C^{\prime}\right)<l$ which contradicts our assumption that $C$ is an odd cycle with the least length in $S(A)$. Therefore, if $m=\min \left(l, l^{\prime}\right)$, then $m=l<l^{\prime}$. We consider the following three subcases.

Subcase 2.1. $x^{\prime} \in C$ and $y^{\prime} \in C$.

Let $w=l\left(P_{1}\right)+l\left(Q_{C}\left(x^{\prime} \rightarrow y^{\prime}\right)\right)+l\left(P_{2}\right)$. If $w$ is odd, set

$$
W^{\prime}=P_{1}+Q_{C}\left(x^{\prime} \longrightarrow y^{\prime}\right)+P_{2}
$$

Otherwise, set

$$
W^{\prime \prime}=P_{1}+C \backslash Q_{C}\left(x^{\prime} \longrightarrow y^{\prime}\right)+P_{2} .
$$

Let

$$
W_{1}=\left\{\begin{array}{ll}
W^{\prime}+C^{1}, & w \text { is odd }, \\
W^{\prime \prime}+C^{1}, & \text { otherwise },
\end{array} \quad W_{2}= \begin{cases}W^{\prime}+C^{2}, & w \text { is odd } \\
W^{\prime \prime}+C^{2}, & \text { otherwise }\end{cases}\right.
$$

Since $C$ is odd, $l\left(Q_{C}\left(x^{\prime} \rightarrow y^{\prime}\right)\right)$ and $l\left(C \backslash Q_{C}\left(x^{\prime} \rightarrow y^{\prime}\right)\right)$ have different parity. Therefore, both $l\left(W_{1}\right)$ and $l\left(W_{2}\right)$ are even. Then, if $w$ is odd,

$$
l\left(W_{1}\right)=l\left(W_{2}\right) \leq 2\left(n-l-l^{\prime}+m\right)+\frac{l-1}{2}+l=2 n-2 l^{\prime}+2 m-\frac{l}{2}-\frac{1}{2} .
$$

Otherwise,

$$
l\left(W_{1}\right)=l\left(W_{2}\right) \leq 2\left(n-l-l^{\prime}+m\right)+l+l=2 n-2 l^{\prime}+2 m .
$$

Since both $l\left(W_{1}\right)$ and $l\left(W_{2}\right)$ are even, it follows that $l\left(W_{1}\right)=l\left(W_{2}\right) \leq 2 n-2$. We see that the pair $W_{1}, W_{2}$ is a pair of SSSD walks with even length. Therefore, there exists a pair of SSSD walks from $x$ to $y$ with length $2 n-2$. 
Subcase 2.2. $x^{\prime} \in C \backslash R$ and $y^{\prime} \in C^{\prime} \backslash R$.

Without loss of generality, we assume that $l\left(Q_{C \backslash R}\left(x^{\prime} \rightarrow v_{1}\right)\right) \leq l\left(Q_{C \backslash R}\left(x^{\prime} \rightarrow v_{m}\right)\right)$. Let $w=l\left(P_{1}\right)+l\left(Q_{C \backslash R}\left(x^{\prime} \rightarrow v_{1}\right)\right)+l\left(Q_{C^{\prime} \backslash R}\left(v_{1} \rightarrow y^{\prime}\right)\right)+l\left(P_{2}\right)$. If $w$ odd, set

$$
W^{\prime}=P_{1}+Q_{C \backslash R}\left(x^{\prime} \longrightarrow v_{1}\right)+Q_{C^{\prime} \backslash R}\left(v_{1} \longrightarrow y^{\prime}\right)+P_{2} .
$$

Otherwise, set

$$
W^{\prime \prime}=P_{1}+Q_{C \backslash R}\left(x^{\prime} \longrightarrow v_{m}\right)+Q_{R}\left(v_{m} \longrightarrow v_{1}\right)+Q_{C^{\prime} \backslash R}\left(v_{1} \longrightarrow y^{\prime}\right)+P_{2}
$$

Let

$$
W_{1}=\left\{\begin{array}{ll}
W^{\prime}+C^{1}, & w \text { is odd }, \\
W^{\prime \prime}+C^{1}, & \text { otherwise },
\end{array} \quad W_{2}= \begin{cases}W^{\prime}+C^{2}, & w \text { is odd } \\
W^{\prime \prime}+C^{2}, & \text { otherwise }\end{cases}\right.
$$

Since $C$ is odd, $l\left(Q_{C \backslash R}\left(x^{\prime} \rightarrow v_{1}\right)\right)$ and $l\left(Q_{C \backslash R}\left(x^{\prime} \rightarrow v_{m}\right)\right)+l\left(Q_{R}\left(v_{m} \rightarrow v_{1}\right)\right)$ have different parity. Therefore, both $l\left(W_{1}\right)$ and $l\left(W_{2}\right)$ are even.

Then, if $w$ is odd,

$$
l\left(W_{1}\right)=l\left(W_{2}\right) \leq 2\left(n-l-l^{\prime}+m\right)+(l-m+1)+\left(l^{\prime}-m+1\right)+l=2 n-l^{\prime}+2 .
$$

Otherwise,

$$
l\left(W_{1}\right)=l\left(W_{2}\right) \leq 2\left(n-l-l^{\prime}+m\right)+(l-m)+\left(l^{\prime}-1\right)+l=2 n-l^{\prime}+m-1 .
$$

Since both $l\left(W_{1}\right)$ and $l\left(W_{2}\right)$ are even, it follows that $l\left(W_{1}\right)=l\left(W_{2}\right) \leq 2 n-2$. We see that the pair $W_{1}, W_{2}$ is a pair of SSSD walks with even length. Therefore, there exists a pair of SSSD walks from $x$ to $y$ with length $2 n-2$.

Subcase 2.3. $x^{\prime} \in C^{\prime} \backslash R$ and $y^{\prime} \in C^{\prime} \backslash R$.

The verification for this subcase is similar to that of the Subcase 2.2 and is omitted.

Therefore, $l(A) \leq 2 n-2$ by Lemma 2.6 , a contradiction.

Lemma 3.5. Let $A$ be an $n \times n$ primitive nonpowerful ZS sign pattern matrix with zero diagonal. If $l(A)=2 n-1$, then $S(A)$ is isomorphic to $G$ (see Figure 1$).$

Proof. Let $A$ be an $n \times n$ primitive nonpowerful ZS sign pattern matrix with zero diagonal. Since $A$ is primitive, it follows from Lemma 2.4 that $S(A)$ is strongly connected and there is an odd cycle $C=\left(v_{1}, v_{2}, \ldots, v_{l}\right)$ in $S(A)$ with length $l(C)=l$. Since $A$ has zero diagonal, there is no loop in $S(A)$ and so $3 \leq l \leq n$. Without loss of generality, we assume that $C$ is an odd cycle with the least length in $S(A)$. If $l(A)=2 n-1$, then by Lemma 3.3, there exists no positive 2-cycle in $S(A)$. Then $S(A)$ is isomorphic to $G$ by Lemma 2.7. We will give anther proof of the theorem.

Denote the vertex set of $S(A)$ by $V$ and the vertex set of $C$ by $V^{\prime}$. By Lemmas 3.4 and 3.3 , the cycle $C$ is the only cycle of length at least 3 in $S(A)$ and there exists no positive 2-cycle 
in $S(A)$. Consider the two directed cycles $C^{1}=\left(v_{1}, v_{2}, \ldots, v_{l}, v_{1}\right)$ and $C^{2}=\left(v_{1}, v_{l}, \ldots, v_{2}, v_{1}\right)$. Since there exists no positive 2-cycle in $S(A)$, the $\operatorname{arcs}\left(v_{i}, v_{i+1}\right)$ and $\left(v_{i+1}, v_{i}\right)$ have different signs. Thus $\operatorname{sgn}\left(C^{1}\right)=-\operatorname{sgn}\left(C^{2}\right)$ by the fact that $l$ is odd. Let $x$ and $y$ be two arbitrary (not necessarily distinct) vertices in $S(A)$. Suppose that $P_{1}$ is the shortest path from $x$ to $C$ and intersects $C$ at vertex $x^{\prime}$ and $P_{2}$ is the shortest path from $y$ to $C$ and intersects $C$ at vertex $y^{\prime}$. If $l=n, S(A)$ is isomorphic to an odd cycle of length $n$. If $l<n$, it is enough to show that there exists a vertex $u \notin V^{\prime}$ in $S(A)$ such that $l(P)=n-l$ where $P$ is the shortest path from $u$ to $C$ and $P$ intersects $C$ at $v$. Suppose not, then

$$
l\left(P_{1}\right), l\left(P_{2}\right) \leq n-l-1 .
$$

Now we consider the following three cases.

Case 1. $x \in V \backslash V^{\prime}$ and $y \in V \backslash V^{\prime}$.

Let $\left.w=l\left(P_{1}\right)+l\left(Q_{C}\left(x^{\prime} \rightarrow y^{\prime}\right)\right)+l\left(P_{2}\right)\right)$. If $w$ is odd, set

$$
W^{\prime \prime}=P_{1}+Q_{C}\left(x^{\prime} \longrightarrow y^{\prime}\right)+P_{2}
$$

Otherwise, set

$$
W^{\prime}=P_{1}+C \backslash Q_{C}\left(x^{\prime} \longrightarrow y^{\prime}\right)+P_{2}
$$

Let

$$
W_{1}=\left\{\begin{array}{ll}
W^{\prime}+C^{1}, & w \text { is odd }, \\
W^{\prime \prime}+C^{1}, & \text { otherwise },
\end{array} \quad W_{2}= \begin{cases}W^{\prime}+C^{2}, & w \text { is odd } \\
W^{\prime \prime}+C^{2}, & \text { otherwise }\end{cases}\right.
$$

Since $C$ is odd, $Q_{C}\left(x^{\prime} \rightarrow y^{\prime}\right)$ and $C \backslash Q_{C}\left(x^{\prime} \rightarrow y^{\prime}\right)$ have different parity. Therefore, both $l\left(W_{1}\right)$ and $l\left(W_{2}\right)$ are even. Then, if $w$ is odd,

$$
l\left(W_{1}\right)=l\left(W_{2}\right) \leq 2(n-l-1)+\frac{l-1}{2}+l=2 n-\frac{l}{2}-\frac{5}{2} .
$$

Otherwise,

$$
l\left(W_{1}\right)=l\left(W_{2}\right) \leq 2(n-l-1)+l+l=2 n-2 .
$$

Since both $l\left(W_{1}\right)$ and $l\left(W_{2}\right)$ are even, it follows that $l\left(W_{1}\right)=l\left(W_{2}\right) \leq 2 n-2$. We see that the pair $W_{1}, W_{2}$ is a pair of SSSD walks with even length. Therefore, there exists a pair of SSSD walks from $x$ to $y$ with length $2 n-2$.

Case 2. $x \in V \backslash V^{\prime}$ and $y \in V$.

In this case, $y^{\prime}=y$. Let $w=l\left(P_{1}\right)+l\left(Q_{C}\left(x^{\prime} \rightarrow y\right)\right)$. If $w$ is odd, set

$$
W^{\prime \prime}=P_{1}+Q_{C}\left(x^{\prime} \longrightarrow y\right) .
$$


Otherwise, set

$$
W^{\prime}=P_{1}+C \backslash Q_{C}\left(x^{\prime} \longrightarrow y\right)
$$

Let

$$
W_{1}=\left\{\begin{array}{ll}
W^{\prime}+C^{1}, & w \text { is odd }, \\
W^{\prime \prime}+C^{1}, & \text { otherwise },
\end{array} \quad W_{2}= \begin{cases}W^{\prime}+C^{2}, & w \text { is odd } \\
W^{\prime \prime}+C^{2}, & \text { otherwise }\end{cases}\right.
$$

Since $C$ is odd, $Q_{C}\left(x^{\prime} \rightarrow y\right)$ and $C \backslash Q_{C}\left(x^{\prime} \rightarrow y\right)$ have different parity. Therefore, both $l\left(W_{1}\right)$ and $l\left(W_{2}\right)$ are even. Then, if $w$ is odd,

$$
l\left(W_{1}\right)=l\left(W_{2}\right) \leq(n-l-1)+\frac{l-1}{2}+l=n+\frac{l}{2}-\frac{3}{2} .
$$

Otherwise,

$$
l\left(W_{1}\right)=l\left(W_{2}\right) \leq(n-l-1)+l+l=n+l-1 .
$$

Since both $l\left(W_{1}\right)$ and $l\left(W_{2}\right)$ are even, it follows that $l\left(W_{1}\right)=l\left(W_{2}\right) \leq 2 n-2$. We see that the pair $W_{1}, W_{2}$ is a pair of SSSD walks with even length. Therefore, there exists a pair of SSSD walks from $x$ to $y$ with length $2 n-2$.

Case 3. $x \in V$ and $y \in V$.

In this case, $x^{\prime}=x$ and $y^{\prime}=y$. Let $w=Q_{C}(x \rightarrow y)$. If $w$ is odd, set

$$
W^{\prime \prime}=Q_{C}(x \longrightarrow y) .
$$

Otherwise, set

$$
W^{\prime}=C \backslash Q_{C}(x \longrightarrow y)
$$

Let

$$
W_{1}=\left\{\begin{array}{ll}
W^{\prime}+C^{1}, & w \text { is odd }, \\
W^{\prime \prime}+C^{1}, & \text { otherwise }
\end{array} \quad W_{2}= \begin{cases}W^{\prime}+C^{2}, & w \text { is odd } \\
W^{\prime \prime}+C^{2}, & \text { otherwise }\end{cases}\right.
$$

Since $C$ is odd, $Q_{C}(x \rightarrow y)$ and $C \backslash Q_{C}(x \rightarrow y)$ have different parity. Therefore, both $l\left(W_{1}\right)$ and $l\left(W_{2}\right)$ are even. Then, if $w$ is odd,

$$
l\left(W_{1}\right)=l\left(W_{2}\right) \leq \frac{l-1}{2}+l=\frac{3 l}{2}-\frac{1}{2} .
$$

Otherwise,

$$
l\left(W_{1}\right)=l\left(W_{2}\right) \leq l+l=2 l .
$$


Since both $l\left(W_{1}\right)$ and $l\left(W_{2}\right)$ are even, it follows that $l\left(W_{1}\right)=l\left(W_{2}\right) \leq 2 n-2$. We see that the pair $W_{1}, W_{2}$ is a pair of SSSD walks with even length. Therefore, there exists a pair of SSSD walks from $x$ to $y$ with length $2 n-2$.

From all the above cases, there exists a pair of SSSD walks from $x$ to $y$ with length $2 n-2$, therefore, $l(A) \leq 2 n-2$ by Lemma 2.6 , a contradiction.

Proof of Theorem 1.6. We get $l(A) \leq 2 n-1$ from Theorem 2.2. Suppose that $A$ is nonpowerful and skew symmetric and $S(A)$ is isomorphic to $G$. Then $l(A)=2 n-1$ by Lemma 2.7.

Conversely, suppose that $l(A)=2 n-1$. We need to prove that $A$ is nonpowerful and skew symmetric and $S(A)$ is isomorphic to $G$. In [1], Li et al. showed that if an irreducible sign pattern matrix $A$ is powerful, then $l(A)=l(|A|)$. Therefore, if $A$ is powerful, then $l(A) \leq 2 n-4$ by Theorem 2.1, which contradicts $l(A)=2 n-1$. Hence $A$ is nonpowerful. Consequently, $A$ is skew symmetric by Lemma 3.3 and $S(A)$ is isomorphic to $G$ by Lemma 3.5.

\section{Acknowledgments}

This paper is supported by NSFC (61170311), Chinese Universities Specialized Research Fund for the Doctoral Program (20110185110020), and Sichuan Province Sci. \& Tech. Research Project (12ZC1802).

\section{References}

[1] Z. Li, F. Hall, and C. Eschenbach, "On the period and base of a sign pattern matrix," Linear Algebra and Its Applications, vol. 212-213, pp. 101-120, 1994.

[2] B. Cheng and B. Liu, "Primitive zero-symmetric sign pattern matrices with the maximum base," Linear Algebra and Its Applications, vol. 433, no. 2, pp. 365-379, 2010.

[3] B. Cheng and B. Liu, "The base sets of primitive zero-symmetric sign pattern matrices," Linear Algebra and Its Applications, vol. 428, no. 4, pp. 715-731, 2008.

[4] Y. Gao, Y. Shao, and J. Shen, "Bounds on the local bases of primitive nonpowerful nearly reducible sign patterns," Linear and Multilinear Algebra, vol. 57, no. 2, pp. 205-215, 2009.

[5] Z. Li, F. J. Hall, and J. L. Stuart, "Irreducible powerful ray pattern matrices," Linear Algebra and Its Applications, vol. 342, no. 1-3, pp. 47-58, 2002.

[6] Z. Li, F. J. Hall, and J. L. Stuart, "Reducible powerful ray pattern matrices," Linear Algebra and Its Applications, vol. 399, no. 1-3, pp. 125-140, 2005.

[7] Y. Liang, B. Liu, and H. J. Lai, "Multi-g base index of primitive anti-symmetric sign pattern matrices," Linear and Multilinear Algebra, vol. 57, no. 6, pp. 535-546, 2009.

[8] B. Liu, B. D. McKay, N. C. Wormald, and Z. K. Min, "The exponent set of symmetric primitive $(0,1)$ matrices with zero trace," Linear Algebra and Its Applications, vol. 133, pp. 121-131, 1990.

[9] B. Liu and L. You, "Bounds on the base of primitive nearly reducible sign pattern matrices," Linear Algebra and Its Applications, vol. 418, no. 2-3, pp. 863-881, 2006.

[10] S. Wang, J. Li, W. Han, and S. Lin, "The base sets of quasi-primitive zero-symmetric sign pattern matrices with zero trace," Linear Algebra and Its Applications, vol. 433, no. 3, pp. 595-605, 2010.

[11] J. Stuart, C. Eschenbach, and S. Kirkland, "Irreducible sign k-potent sign pattern matrices," Linear Algebra and Its Applications, vol. 294, no. 1-3, pp. 85-92, 1999.

[12] L. You, J. Shao, and H. Shan, "Bounds on the bases of irreducible generalized sign pattern matrices," Linear Algebra and Its Applications, vol. 427, no. 2-3, pp. 285-300, 2007.

[13] R. A. Brualdi and H. J. Ryser, Combinatorial Matrix Theory, vol. 39 of Encyclopedia of Mathematics and Its Applications, Cambridge University Press, Cambridge, UK, 1991. 


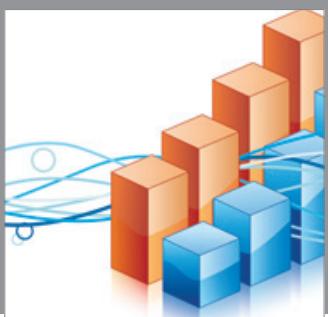

Advances in

Operations Research

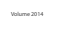

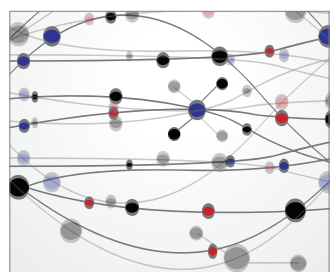

\section{The Scientific} World Journal
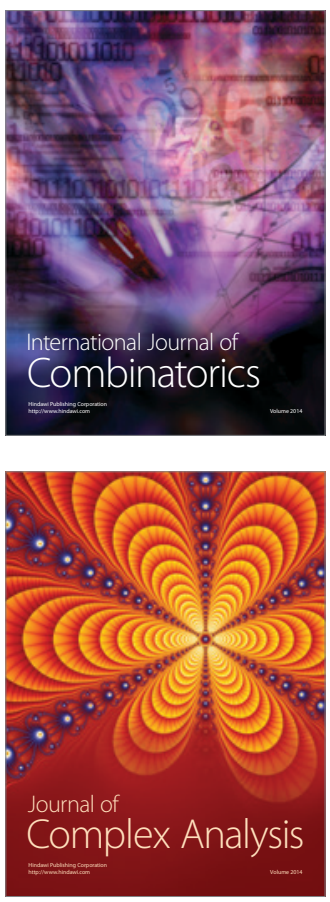

International Journal of

Mathematics and

Mathematical

Sciences
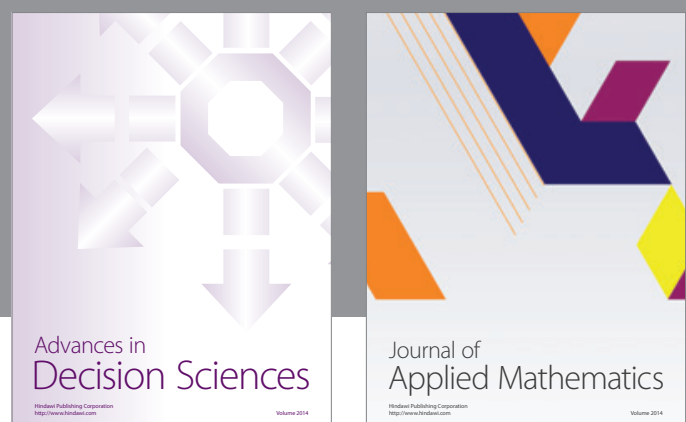

Journal of

Applied Mathematics
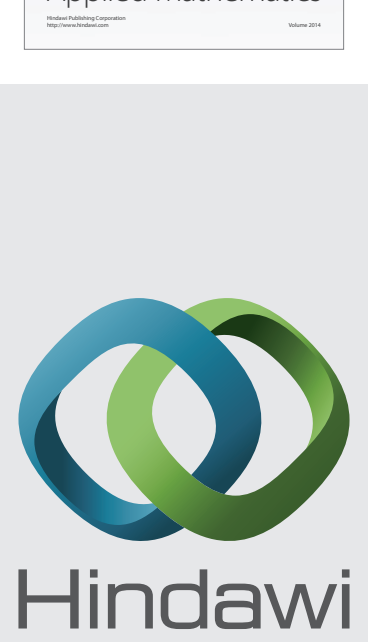

Submit your manuscripts at http://www.hindawi.com
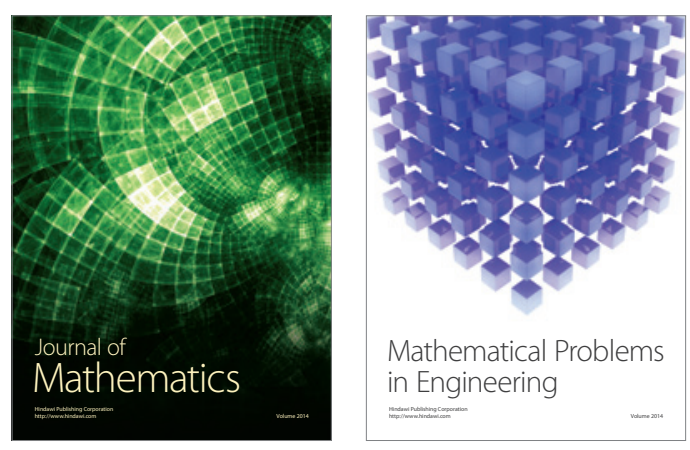

Mathematical Problems in Engineering
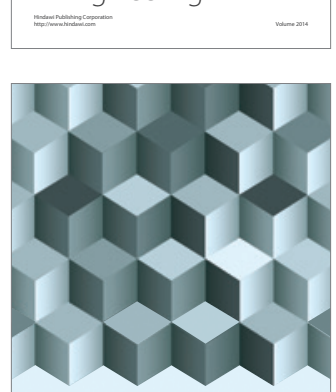

Journal of

Function Spaces
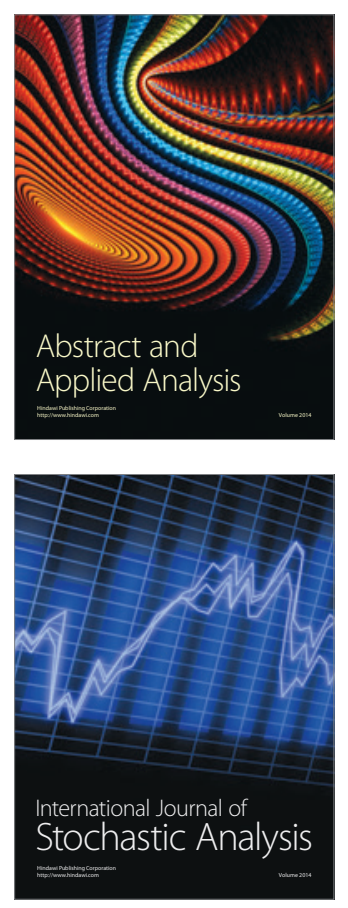

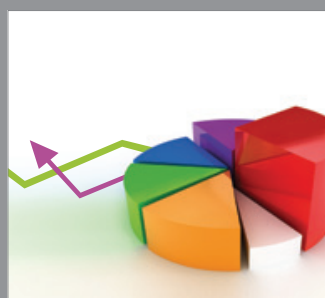

ournal of

Probability and Statistics

Promensencen
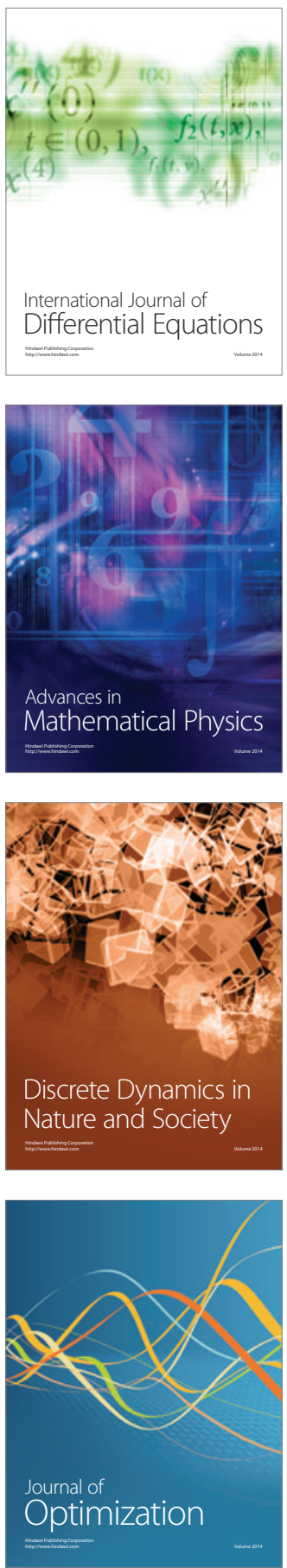